\title{
Existence of Invariant Curves for Degenerate Quasi-periodic Reversible Mappings
}

\section{Peng Huang}

\begin{abstract}
In this paper we are concerned with the existence of invariant curves of quasi-periodic reversible mappings with higher order degeneracy of the twist condition under the Brjuno-Rüssmann's non-resonant condition. In the proof we use a new variant of the KAM theory, containing an artificial parameter $q, 0<q<1$, which makes the steps of the KAM iteration infinitely small in the speed of function $q^{n} \varepsilon$, rather than super exponential function.
\end{abstract}

\section{Introduction}

In this paper we are concerned with the existence of invariant curves of the following planar quasi-periodic mapping

$$
\mathcal{A}:\left\{\begin{array}{l}
x_{1}=x+h(y)+f(x, y) \\
y_{1}=y+g(x, y)
\end{array}\right.
$$

where the frequency map $h(y)$ is real analytic, the small perturbations $f(x, y)$ and $g(x, y)$ are quasi-periodic (see Definition 2.1) in $x$ with frequencies $\omega=\left(\omega_{1}, \omega_{2}, \ldots, \omega_{m}\right.$ ), real analytic in $x$ and $y$, the variable $y$ ranges in an open interval $I \subseteq \mathbb{R}$. The mapping is called area-preserving mapping, if it preserves area. Correspondingly, the mapping is called reversible mapping with respect to the involution $\mathcal{R}:(x, y) \mapsto(-x, y)$, if $\mathcal{A} \mathcal{R} \mathcal{A}=\mathcal{R}$. Our main concern will be the analytic quasi-periodic reversible mapping $\mathcal{A}$ with higher order degeneracy of the twist condition, that is, there is some value $y_{0} \in I$ such that $h^{\prime}\left(y_{0}\right)=0, \ldots, h^{(\lambda-1)}\left(y_{0}\right)=0, h^{(\lambda)}\left(y_{0}\right) \neq 0$ with $\lambda \in \mathbb{Z}>1$.

When the perturbations $f=g=0$ in $(1.1)$, the mapping $\mathcal{A}$ has a family of invariant curves with $h(y)$ as its rotation number. The condition

$$
\frac{d h(y)}{d y} \neq 0 \quad \text { for all } y \in I
$$

Received October 20, 2021; Accepted February 11, 2022.

Communicated by Cheng-Hsiung Hsu.

2020 Mathematics Subject Classification. 37J40, 70K43.

Key words and phrases. invariant curves, quasi-periodic reversible mappings, higher order degeneracy of the twist condition, Brjuno-Rüssmann's non-resonant condition, KAM theory.

This pape was partially supported by the National Natural Science Foundation of China (11901131), Guizhou Provincial Science and Technology Foundation ([2020]1Y006). 
is called twist condition. Under this condition the mapping (1.1) is called twist mapping and can be equivalent to

$$
\mathcal{A}_{0}:\left\{\begin{array}{l}
x_{1}=x+y+f(x, y) \\
y_{1}=y+g(x, y)
\end{array}\right.
$$

Moser [21] considered the area-preserving twist mapping

$$
\mathfrak{M}_{0}:\left\{\begin{array}{l}
x_{1}=x+\alpha(y)+\varphi_{1}(x, y) \\
y_{1}=y+\varphi_{2}(x, y)
\end{array}\right.
$$

where the perturbations $\varphi_{1}, \varphi_{2}$ are assumed to be small and of period $2 \pi$ in $x$. He obtained the existence of invariant closed curves of $\mathfrak{M}_{0}$ which is of class $\mathcal{C}^{333}$. About $\mathfrak{M}_{0}$, an analytic version of the invariant curve theorem was presented in [30], a version in class $\mathcal{C}^{5}$ in Rüssmann [27] and a optimal version in class $\mathcal{C}^{p}$ with $p>3$ in Herman 9 . 10 .

When the perturbations $f(x, y), g(x, y)$ in 1.3 are quasi-periodic in $x$, there are some results about the existence of invariant curves of the following planar quasi-periodic twist mapping

$$
\mathfrak{M}_{1}:\left\{\begin{array}{l}
x_{1}=x+\alpha+y+f(x, y), \\
y_{1}=y+g(x, y),
\end{array} \quad(x, y) \in \mathbb{R} \times[a, b],\right.
$$

where the functions $f(x, y)$ and $g(x, y)$ are quasi-periodic in $x$ with the frequency $\omega=$ $\left(\omega_{1}, \omega_{2}, \ldots, \omega_{m}\right)$, real analytic in $x$ and $y$, and $\alpha$ is a constant.

When the map $\mathfrak{M}_{1}$ in (1.4) is an exact symplectic map, $\omega_{1}, \omega_{2}, \ldots, \omega_{m}, 2 \pi \alpha^{-1}$ are sufficiently incommensurable, Zharnitsky [34] proved the existence of invariant curves of the map $\mathfrak{M}_{1}$ and applied this result to present the boundedness of all solutions of FermiUlam problem. His proof is based on the Lagrangian approach introduced by Moser 24$]$ and used by Levi and Moser in 15 to show a proof of the twist theorem.

When the map $\mathfrak{M}_{1}$ in $(1.4)$ is reversible with respect to the involution $\mathcal{R}:(x, y) \mapsto$ $(-x, y)$, that is, $\mathcal{R M}_{1} \mathcal{R}=\mathfrak{M}_{1}^{-1}, \omega_{1}, \omega_{2}, \ldots, \omega_{m}, 2 \pi \alpha^{-1}$ satisfy the Diophantine condition

$$
\left|\langle k, \omega\rangle \frac{\alpha}{2 \pi}-j\right| \geq \frac{\gamma}{|k|^{\tau}} \text { for all } k \in \mathbb{Z}^{m} \backslash\{0\}, j \in \mathbb{Z},
$$

Liu [17] obtained some variants of the invariant curve theorem for quasi-periodic reversible mapping $\mathfrak{M}_{1}$. As an application, he used the invariant curve theorem to investigate the existence of quasi-periodic solutions and the boundedness of all solutions for an asymmetric oscillator depending quasi-periodically on time. On the other hand, by establishing the invariant curve theorem of planar smooth quasi-periodic twist mappings in [12], recently we obtained the existence of quasi-periodic solutions and the boundedness of all solutions for an asymmetric oscillation with a quasi-periodic external force 13 . 
For the reversible mapping, the invariant curve theorem was obtained by Moser 22, 23], then developed by Sevryuk [29]. Liu [18] concerned with the existence of invariant curves of reversible mappings, and obtained a variants of the small twist theorem. In the previous proofs in 17, 18, 21, 34], the steps of KAM iteration decay in speed of super exponential function $\varepsilon^{\mu^{n}}, 1<\mu<2$.

After the research of the twist mapping, it is clear that an important direction of further investigation is the non-twist mapping which violates the twist condition 1.2 , that is, there is some $y_{0}$ such that $h^{\prime}\left(y_{0}\right)=0$.

When the perturbations $f(x, y), g(x, y)$ in 11.1 are $2 \pi$-periodic in $x$, Simó 31 proved the existence of invariant curves for the periodic non-twist area preserving mapping. For other well known results on the periodic non-twist area preserving, refer to [1, 3, 6 and the references therein. As we know if the twist condition is not satisfied, the frequency of invariant curves may have some drift rather than the frequency remain unchanged. However, in the above references, for the periodic non-twist mapping one can only obtain the existence of invariant curves, there is no information about the frequency of the invariant curves.

Cheng and Xia [2] proved a generalization to the classical KAM theorem, replacing the standard twist or nondegenerate condition with a weaker topological condition. This generalization was also proved by Herman 11 .

Zhang and $\mathrm{Xu}$ [33] obtained the invariant curve theorem for the analytic periodic reversible non-twist mapping under the Brjuno-Rüssmann's non-resonant condition. They mainly use the ideas in [26, 28], introduce a parameter $q, 0<q<1$, and make the steps of the KAM-iteration infinitely small in the speed of function $q^{n} \varepsilon$, rather than super exponential function $\varepsilon^{\mu^{n}}, 1<\mu<2$. Moreover, they also investigated the persistence of the frequency of the invariant curves.

The study of the existence of invariant curves of area preserving non-twist maps, and equivalently in degenerate Hamiltonian systems, is a problem of both theoretical and practical relevance. Recently, there has been considerable interest in studying the persistence of tori of Hamiltonian system with fixed frequency, where twist condition is violated but nevertheless, the system depends on sufficient parameters that control the frequencies. González-Enríquez, Haro and Llave [8, Chapter 9] obtain persistence of an invariant torus with fixed frequency in both the twist and the non-twist cases, including small twist.

In this paper we focus on the quasi-periodic case, that is, the perturbations $f(x, y)$, $g(x, y)$ in (1.1) are quasi-periodic periodic in $x$ with the frequencies $\omega=\left(\omega_{1}, \omega_{2}, \ldots, \omega_{m}\right)$, and similar to $17,18,33$, we also assume that the mapping $\mathcal{A}$ in (1.1) is reversible with respect to the involution $\mathcal{R}:(x, y) \mapsto(-x, y)$, that is, $\mathcal{A R} \mathcal{A}=\mathcal{R}$, instead of the exact 
symplecticity or area-preserving assumption on $\mathcal{A}$, and want to establish the invariant curve theorem for the quasi-periodic mapping $\mathcal{A}$ with higher order degeneracy of the twist condition, that is, there is some value $y_{0} \in I$ such that $h^{\prime}\left(y_{0}\right)=0, \ldots, h^{(\lambda-1)}\left(y_{0}\right)=$ $0, h^{(\lambda)}\left(y_{0}\right) \neq 0$ with $\lambda \in \mathbb{Z}>1$. However, we remark that unlike the standard KAM theorems where the invariant curve for any fixed diophantine rotation number is unique, here we may have many or even infinitely many invariant curves with the same rotation numbers. For instance, in (1.1) if $h(y)=\beta^{*}+y^{3}$ and $f(x, y)=\varepsilon y\left(\sin \omega_{1} x+\sin \omega_{2} x+\right.$ $\left.\cdots+\sin \omega_{m} x\right), g(x, y)=0$, then for any $\beta$ close to $\beta^{*}$ there are three invariant curves with rotation number $\beta$.

As in 25, 32, 33, we introduce some parameters, so that the existence of invariant curves of quasi-periodic mapping $\mathcal{A}$ in 1.1 can be reduced to that of a family of quasi-periodic mappings with some parameters. We write $y=p+z$, and expand $h(y)$ around $p$ such that $h(y)=h(p)+\int_{0}^{1} h^{\prime}\left(y_{t}\right) z d t$, where $y_{t}=p+t z, 0 \leq t \leq 1, z$ varies in a neighborhood of origin of the real line $\mathbb{R}$. We put $\Omega(p)=h(p), f(x, z ; p)=\int_{0}^{1} h^{\prime}\left(y_{t}\right) z d t+f(x, p+z)$, $g(x, z ; p)=g(x, p+z)$, and obtain the family of analytic quasi-periodic reversible mappings

$$
\mathfrak{M}:\left\{\begin{array}{l}
x_{1}=x+\Omega(p)+f(x, z ; p), \\
z_{1}=z+g(x, z ; p)
\end{array}\right.
$$

Now we turn to consider this family of analytic quasi-periodic reversible mappings $\mathfrak{M}$ with parameters $p \in \Pi$ such that $\Omega(p) \neq 0$ and $\Pi \subseteq I$ is a neighborhood of $y_{0}$, which is a bounded interval such as $\left(y_{0}-\delta, y_{0}+\delta\right), \delta>0$.

In this paper, under the Brjuno-Rüssmann's non-resonant condition (2.3), the existence of invariant curves of analytic quasi-periodic reversible mappings $\mathfrak{M}$ will be proved. In the proof we use a new variant of the KAM theory in [26, 28, 33], containing an artificial parameter $q, 0<q<1$, which makes the steps of the KAM iteration infinitely small in the speed of function $q^{n} \varepsilon$, rather than super exponential function. Moreover, we will investigate the frequency of the invariant curves, the frequency can not be regarded as independent parameters. The frequency may have some drifts at each step, rather than the frequencies remain unchanged. Hence, we can also obtain the existence of the invariant curves for the analytic quasi-periodic reversible mappings $\mathcal{A}$.

After we get the invariant curve theorem, as an application, we shall study the existence of quasi-periodic solutions whose frequencies are not independent parameters for the following superlinear Duffing's equation

$$
\ddot{x}+x^{3}=f(t),
$$

where $f(t)$ is real analytic and quasi-periodic in $t$ with the frequency $\omega=\left(\omega_{1}, \omega_{2}, \ldots, \omega_{m}\right)$. 
It is well known that the longtime behaviour of a time dependent nonlinear differential equation

$$
\ddot{x}+f(t, x)=0
$$

$f$ being periodic in $t$, can be very intricate. For example, there are equations having unbounded solutions but with infinitely many zeros and with nearby unbounded solution having randomly prescribed numbers of zeros and also periodic solution (see [7]).

In contrast to such unboundedness phenomena one may look for conditions on the nonlinearity, in addition to the superlinear condition that

$$
\frac{1}{x} f(t, x) \rightarrow \infty \quad \text { as }|x| \rightarrow \infty,
$$

which allow to conclude that all solutions of equation (1.7) are bounded. For example, every solution of equation (1.6) with $p(t+1)=p(t)$ being continuous, is bounded. This result, prompted by Littlewood in [16], is due to Morris [20], who proved that there are infinitely many quasi-periodic solutions and the boundedness of all solutions of (1.6). In 1987, Dieckerhoff and Zehnder in [7] extended this result to the general superlinear Duffing's equation. For recent development, we refer to [14, 19] and the references therein.

The rest of the paper is organized as follows. In Section 2, we list some properties of quasi-periodic functions, and then state the main invariant curve theorem (see Theorem 2.7) for the quasi-periodic reversible mappings $\mathfrak{M}$ which is defined by (1.5). We introduce some tools which will be used to prove the invariant curve theorem, and give the proof of Theorem 2.7 in Section 3. Some examples and applications are given in Section 4 .

\section{Main result}

2.1. The space of quasi-periodic functions

We first define the space of real analytic quasi-periodic functions $Q(\omega)$ as in [30, Chapter 3]. Here the $m$-dimensional frequency vector $\omega=\left(\omega_{1}, \omega_{2}, \ldots, \omega_{m}\right)$ is rationally independent, that is, for all $k=\left(k_{1}, k_{2}, \ldots, k_{m}\right) \neq 0,\langle k, \omega\rangle=\sum_{j} k_{j} \omega_{j} \neq 0$.

Definition 2.1. A function $f: \mathbb{R} \rightarrow \mathbb{R}$ is called real analytic quasi-periodic with the frequency $\omega=\left(\omega_{1}, \omega_{2}, \ldots, \omega_{m}\right)$, if there exists a real analytic function

$$
F: \theta=\left(\theta_{1}, \theta_{2}, \ldots, \theta_{m}\right) \in \mathbb{R}^{m} \rightarrow \mathbb{R}
$$

such that $f(t)=F\left(\omega_{1} t, \omega_{2} t, \ldots, \omega_{m} t\right)$ for all $t \in \mathbb{R}$, where $F$ is $2 \pi$-periodic in each variable and bounded in a complex neighborhood $\Pi_{r}^{m}=\left\{\left(\theta_{1}, \theta_{2}, \ldots, \theta_{m}\right) \in \mathbb{C}^{m}:\left|\operatorname{Im} \theta_{j}\right| \leq r, j=\right.$ $1,2, \ldots, m\}$ of $\mathbb{R}^{m}$ for some $r>0$. Here we call $F(\theta)$ the shell function of $f(t)$. 
Denote by $Q(\omega)$ the set of real analytic quasi-periodic functions with the frequency $\omega=\left(\omega_{1}, \omega_{2}, \ldots, \omega_{m}\right)$. Given $f(t) \in Q(\omega)$, the shell function $F(\theta)$ of $f(t)$ admits a Fourier series expansion

$$
F(\theta)=\sum_{k \in \mathbb{Z}^{m}} f_{k} e^{i\langle k, \theta\rangle}
$$

where $k=\left(k_{1}, k_{2}, \ldots, k_{m}\right), k_{j}$ range over all integers and the coefficients $f_{k}$ decay exponentially with $|k|=\left|k_{1}\right|+\left|k_{2}\right|+\cdots+\left|k_{m}\right|$, then $f(t)$ can be represented as a Fourier series of the type from the definition

$$
f(t)=\sum_{k \in \mathbb{Z}^{m}} f_{k} e^{i\langle k, \omega\rangle t} .
$$

In the following we define the norm of the real analytic quasi-periodic function $f(t)$ through that of the corresponding shell functions $F$.

Definition 2.2. For $r>0$, let $Q_{r}(\omega) \subseteq Q(\omega)$ be the set of real analytic quasi-periodic functions $f$ such that the corresponding shell functions $F$ are bounded on the subset $\Pi_{r}^{m}$ with the supremum norm

$$
|F|_{r}=\sup _{\theta \in \Pi_{r}^{m}}|F(\theta)|=\sup _{\theta \in \Pi_{r}^{m}}\left|\sum_{k} f_{k} e^{i\langle k, \theta\rangle}\right|<+\infty .
$$

Thus we define $|f|_{r}:=|F|_{r}$.

The following properties of real analytic quasi-periodic functions can be found in 30 , Chapter 3].

Lemma 2.3. The following statements are true:

(i) Let $f(t), g(t) \in Q(\omega)$, then $g(t+f(t)) \in Q(\omega)$;

(ii) Suppose that

$$
|\langle k, \omega\rangle| \geq \frac{c}{|k|^{\sigma_{0}}}, \quad c, \sigma_{0}>0
$$

for all integer vectors $k \neq 0$. Let $h(t) \in Q(\omega)$ and $\tau=\beta t+h(t)\left(\beta+h^{\prime}>0\right)$, then the inverse relation is given by $t=\beta^{-1} \tau+h_{1}(\tau)$ and $h_{1} \in Q(\omega / \beta)$. In particular, if $\beta=1$, then $h_{1} \in Q(\omega)$.

Throughout this paper, we assume that the frequency $\omega=\left(\omega_{1}, \omega_{2}, \ldots, \omega_{m}\right)$ satisfies the Diophantine condition

$$
|\langle k, \omega\rangle| \geq \frac{c}{|k|^{\sigma_{0}}}, \quad c, \sigma_{0}>0
$$

for all integer vectors $k \neq 0$. It is not difficult to show that for $\sigma_{0}>m-1$, the Lebesgue measure of the set of $\omega$ satisfying the above inequalities is positive for a suitably small $c$. Let $|\omega|_{\infty}=\max _{1 \leq i \leq m}\left|\omega_{i}\right|$. 


\subsection{The Brjuno-Rüssmann's non-resonant condition}

Since there is some value $y_{0} \in I$ such that

$$
h^{\prime}\left(y_{0}\right)=0, \ldots, h^{(\lambda-1)}\left(y_{0}\right)=0, h^{(\lambda)}\left(y_{0}\right) \neq 0
$$

with $\lambda \in \mathbb{Z}>1$, as a matter of fact, it is apparent that there exist a bounded interval $\Pi$ such that $\Omega(p)=h(p)$ satisfies

$$
\Omega^{(\lambda)}(p) \neq 0, \quad \lambda \in \mathbb{Z}>1 \quad \text { for all } p \in \Pi,
$$

where $\Pi \subseteq I$ is a neighborhood of $y_{0}$, which is a bounded interval such as $\left(y_{0}-\delta, y_{0}+\delta\right)$, $\delta>0$.

In KAM step, $\Omega(p)$ also need satisfy the Brjuno-Rüssmann's non-resonant condition

$$
\left|\langle k, \omega\rangle \frac{\Omega(p)}{2 \pi}-j\right| \geq \frac{\gamma}{\Delta(|k|)} \quad \text { for all } k \in \mathbb{Z}^{m} \backslash\{0\}, j \in \mathbb{Z}
$$

with some $\gamma>0$ and Brjuno-Rüssmann approximation function $\Delta$. We call $\Delta:[1, \infty) \rightarrow$ $[1, \infty)$ an Brjuno-Rüssmann approximation function, that means it is continuous, increasing, unbounded such that $\Delta(1)=1$ and

$$
\int_{1}^{\infty} \frac{\ln \Delta(t)}{t^{2}} d t<\infty
$$

Remark 2.4. If we choose $\Delta(t)=t^{\mu}$, the Brjuno-Rüssmann's non-resonant condition (2.3) becomes the Diophantine condition which had been used in [17, 21, 34.

In the following the measure estimate of the parameters $p$ such that the frequency $\Omega(p)$ satisfies the Brjuno-Rüssmann's non-resonant condition (2.3) under $(2.2)$ will be given.

Theorem 2.5. If the Brjuno-Rüssmann approximation function $\Delta$ is chosen such that

$$
\sum_{\ell=1}^{+\infty} \ell^{m}\left(\frac{1}{\ell \Delta(\ell)}\right)^{1 / \lambda}<+\infty
$$

then for suitable $\gamma$, the set of $p$ satisfying the Brjuno-Rüssmann's non-resonant condition (2.3) under 2.2 has positive measure.

Proof. Choose some $m$-dimensional frequency vector $\omega=\left(\omega_{1}, \omega_{2}, \ldots, \omega_{m}\right)$ satisfying (2.1) and let $\Pi_{\omega, \gamma, \Delta}$ denote the set of all $p \in \Pi$ satisfying $(2.3)$ with the fixed $\omega, \gamma, \Delta$. Then $\Pi_{\omega, \gamma, \Delta}$ is the complement of the open dense set $R_{\omega, \gamma, \Delta}$, where

$$
\mathrm{R}_{\omega, \gamma, \Delta}=\bigcup_{\substack{k \in \mathbb{Z}^{m} \backslash\{0\} \\ j \in \mathbb{Z}}} \mathrm{R}_{\omega, \gamma, \Delta}^{k, j}=\bigcup_{\substack{k \in \mathbb{Z}^{m} \backslash\{0\} \\ j \in \mathbb{Z}}}\left\{p \in \Pi:\left|\langle k, \omega\rangle \frac{\Omega(p)}{2 \pi}-j\right|<\frac{\gamma}{\Delta(|k|)}\right\} .
$$


Now we estimate the measure of the set $\mathrm{R}_{\omega, \gamma, \Delta}^{k, j}$. Set $\left|k_{\max }\right|=\max _{1 \leq i \leq m}\left|k_{i}\right|$, then there exists some $1 \leq \widetilde{m} \leq m$ such that $\left|k_{\tilde{m}}\right|=\left|k_{\max }\right|$, and $1 \leq|k| /\left|k_{\max }\right| \leq m$. Therefore, we have

$$
\begin{aligned}
\mathrm{R}_{\omega, \gamma, \Delta}^{k, j} & =\left\{p \in \Pi:\left|\langle k, \omega\rangle \frac{\Omega(p)}{2 \pi}-j\right|<\frac{\gamma}{\Delta(|k|)}\right\} \\
& =\left\{p \in \Pi:\left|k_{\max } \omega_{\widetilde{m}} \frac{\Omega(p)}{2 \pi}+\sum_{i \neq \widetilde{m}} k_{i} \omega_{i} \frac{\Omega(p)}{2 \pi}-j\right|<\frac{\gamma}{\Delta(|k|)}\right\} .
\end{aligned}
$$

Since there is some value $y_{0}$ such that $h^{\prime}\left(y_{0}\right)=0, \ldots, h^{(\lambda-1)}\left(y_{0}\right)=0, h^{(\lambda)}\left(y_{0}\right) \neq 0$ with $\lambda>1$, then

$$
\Omega^{(i)}\left(y_{0}\right)=h^{(i)}\left(y_{0}\right)=0, \quad 1 \leq i<\lambda .
$$

And by 2.2, we know

$$
\Omega^{(\lambda)}(p) \neq 0 \text { for all } p \in \Pi .
$$

Hence there exists $\zeta \in \Pi$ between $p$ and $y_{0}$ such that $\Omega^{(\lambda)}(\zeta) \neq 0$ and

$$
\Omega(p)=\Omega\left(y_{0}\right)+\frac{\Omega^{(\lambda)}(\zeta)}{\lambda !}\left(p-y_{0}\right)^{\lambda}=h\left(y_{0}\right)+\frac{\Omega^{(\lambda)}(\zeta)}{\lambda !}\left(p^{\lambda}+\sum_{\jmath=1}^{\lambda}(-1)^{\jmath}\left(\begin{array}{l}
\lambda \\
\jmath
\end{array}\right) p^{\lambda-\jmath} y_{0}^{\jmath}\right)
$$

Therefore,

$$
\begin{aligned}
\mathrm{R}_{\omega, \gamma, \Delta}^{k, j} & =\left\{p \in \Pi: \frac{\left|k_{\max }\right|\left|\omega_{\widetilde{m}}\right|\left|\Omega^{(\lambda)}(\zeta)\right|}{\lambda !}\left|p^{\lambda}-b_{j}\right|<\frac{2 \pi \gamma}{\Delta(|k|)}\right\} \\
& =\left\{p \in \Pi: b_{j}-\delta_{k}<|p|^{\lambda}<b_{j}+\delta_{k}\right\},
\end{aligned}
$$

where $b_{j}=-\sum_{\jmath=1}^{\lambda}(-1)^{\jmath}\left(\begin{array}{c}\lambda \\ \jmath\end{array}\right) p^{\lambda-\jmath} y_{0}^{\jmath}-\frac{\lambda !}{\left|k_{\max }\right|\left|\omega_{\tilde{m}}\right|\left|\Omega^{(\lambda)}(\zeta)\right|}\left\{k_{\max } \omega_{\widetilde{m}} h\left(y_{0}\right)+\sum_{i \neq \widetilde{m}} k_{i} \omega_{i} \Omega(p)-\right.$ $2 \pi j\}$ and $\delta_{k}=\frac{2 \pi \gamma}{\Delta(|k|)} \frac{\lambda !}{\left|k_{\max }\right|\left|\omega_{\widetilde{m}}\right|\left|\Omega^{(\lambda)}(\zeta)\right|}$.

If $b_{j}-\delta_{k} \leq 0$ which means $b_{j} \leq \delta_{k}$, then

$$
\begin{aligned}
\operatorname{meas}\left(\mathrm{R}_{\omega, \gamma, \Delta}^{k, j}\right) \leq 2\left(2 \delta_{k}\right)^{1 / \lambda} & =2\left(\frac{4 \pi \gamma}{\Delta(|k|)} \frac{\lambda !}{\left|k_{\max }\right|\left|\omega_{\widetilde{m}}\right|\left|\Omega^{(\lambda)}(\zeta)\right|}\right)^{1 / \lambda} \\
& =2\left(\frac{\gamma}{|k| \Delta(|k|)}\right)^{1 / \lambda}\left(\frac{|k|}{\left|k_{\max }\right|}\right)^{1 / \lambda}\left(\frac{4 \pi \lambda !}{\left|\omega_{\widetilde{m}}\right|\left|\Omega^{(\lambda)}(\zeta)\right|}\right)^{1 / \lambda} .
\end{aligned}
$$

If $b_{j}-\delta_{k}>0$ which means $b_{j}+\delta_{k}>b_{j}-\delta_{k}>0$, and by the inequality $x^{1 / \lambda}-a^{1 / \lambda} \leq$ $(x-a)^{1 / \lambda}$ when $\lambda \geq 1, x \geq a>0$, then

$$
\begin{aligned}
\operatorname{meas}\left(\mathrm{R}_{\omega, \gamma, \Delta}^{k, j}\right) & \leq 2\left(\left(b_{j}+\delta_{k}\right)^{1 / \lambda}-\left(b_{j}-\delta_{k}\right)^{1 / \lambda}\right) \leq 2\left(\left(b_{j}+\delta_{k}\right)-\left(b_{j}-\delta_{k}\right)\right)^{1 / \lambda} \\
& \leq 2\left(2 \delta_{k}\right)^{1 / \lambda}=2\left(\frac{4 \pi \gamma}{\Delta(|k|)} \frac{\lambda !}{\left|k_{\max }\right|\left|\omega_{\tilde{m}}\right|\left|\Omega^{(\lambda)}(\zeta)\right|}\right)^{1 / \lambda} \\
& =2\left(\frac{\gamma}{|k| \Delta(|k|)}\right)^{1 / \lambda}\left(\frac{|k|}{\left|k_{\max }\right|}\right)^{1 / \lambda}\left(\frac{4 \pi \lambda !}{\left|\omega_{\tilde{m}}\right|\left|\Omega^{(\lambda)}(\zeta)\right|}\right)^{1 / \lambda} .
\end{aligned}
$$


Since $1 \leq|k| / k_{\max } \leq m$, then we have the following measure estimate

$$
\operatorname{meas}\left(\mathrm{R}_{\omega, \gamma, \Delta}^{k, j}\right) \leq O\left(\left(\frac{\gamma}{|k| \Delta(|k|)}\right)^{1 / \lambda}\right)
$$

Next we estimate the measure of the set $\mathrm{R}_{\omega, \gamma, \Delta}$. Since $k \in \mathbb{Z}^{m} \backslash\{0\}, j \in \mathbb{Z}$,

$$
\left|\langle k, \omega\rangle \frac{\Omega(p)}{2 \pi}-j\right|<\frac{\gamma}{\Delta(|k|)},
$$

then we have

$$
|j| \leq\left|\langle k, \omega\rangle \frac{\Omega(p)}{2 \pi}\right|+\frac{\gamma}{\Delta(|k|)} \leq c_{0}|k|,
$$

where $c_{0}$ is a constant independent of $k$. Thus

$$
\begin{aligned}
\operatorname{meas}\left(\mathrm{R}_{\omega, \gamma, \Delta}\right) & \leq \sum_{k \in \mathbb{Z}^{m} \backslash\{0\}} \sum_{\substack{j \in \mathbb{Z} \\
|j| \leq c_{0}|k|}} \operatorname{meas}\left(\mathrm{R}_{\omega, \gamma, \Delta}^{k, j}\right) \leq \sum_{k \in \mathbb{Z}^{m} \backslash\{0\}} \sum_{\substack{j \in \mathbb{Z} \\
|j| \leq c_{0}|k|}} O\left(\left(\frac{\gamma}{|k| \Delta(|k|)}\right)^{1 / \lambda}\right) \\
& \leq \sum_{k \in \mathbb{Z}^{m} \backslash\{0\}} O\left(|k|\left(\frac{\gamma}{|k| \Delta(|k|)}\right)^{1 / \lambda}\right) .
\end{aligned}
$$

Also, by the condition of Theorem 2.5, we have

$$
\begin{aligned}
\sum_{k \in \mathbb{Z}^{m} \backslash\{0\}}|k|\left(\frac{1}{|k| \Delta(|k|)}\right)^{1 / \lambda} & \leq 2^{m} \sum_{\ell=1}^{+\infty} \ell\left(\frac{1}{\ell \Delta(\ell)}\right)^{1 / \lambda}\left(\begin{array}{c}
m+\ell-1 \\
\ell
\end{array}\right) \\
& \leq 2^{2 m-1} \sum_{\ell=1}^{+\infty} \ell^{m}\left(\frac{1}{\ell \Delta(\ell)}\right)^{1 / \lambda}<+\infty
\end{aligned}
$$

Hence

$$
\operatorname{meas}\left(\mathrm{R}_{\omega, \gamma, \Delta}\right) \leq O\left(\gamma^{1 / \lambda}\right) \quad \text { and } \quad \operatorname{meas}\left(\Pi_{\omega, \gamma, \Delta}\right) \rightarrow \operatorname{meas}(\Pi) \quad \text { as } \gamma \rightarrow 0
$$

This completes the proof of Theorem 2.5.

Remark 2.6. From power function $t^{\beta}, \beta>\lambda m+\lambda-1$, to exponent function $e^{t^{1-} \tilde{\gamma}}, \widetilde{\gamma}<1$, can all be chosen as a Brjuno-Rüssmann approximation function.

Throughout this paper, we assume that the Brjuno-Rüssmann approximation function $\Delta$ has been chosen such that Theorem 2.5 is established. Hence $\Pi_{\omega, \gamma, \Delta}$ the set of all $p \in \Pi$ satisfying (2.3) under (2.2) has positive measure. Finally, with any approximation function $\Delta$ we define another function $\Lambda$ by setting $\Lambda(t)=t \Delta(t)$. 


\subsection{The main result}

Before stating our theorem, we first give some definitions and notations. Define

$$
D(r, s)=\left\{(\theta, z) \in \mathbb{C}^{m} \times \mathbb{C}:\left|\operatorname{Im} \theta_{j}\right| \leq r, j=1,2, \ldots, m,|z| \leq s\right\}
$$

and a complex neighborhood of $\Pi_{\omega, \gamma, \Delta}$,

$$
\Pi_{h}=\left\{p \in \mathbb{C}: \operatorname{dist}\left(p, \Pi_{\omega, \gamma, \Delta}\right) \leq h\right\}
$$

Suppose the function $f(x, z ; p)$ is quasi-periodic in $x$ with the frequency $\omega=\left(\omega_{1}, \omega_{2}\right.$, $\left.\ldots, \omega_{m}\right)$ and real analytic on $D(r, s) \times \Pi_{h}$. We expand $f(x, z ; p)$ as Fourier series with respect to $x$,

$$
f(x, z ; p)=\sum_{k \in \mathbb{Z}^{m}} f_{k}(z ; p) e^{i\langle k, \omega\rangle x}
$$

then define

$$
\|f\|_{r, s, h}=\sum_{k \in \mathbb{Z}^{m}}\left|f_{k}\right|_{s, h} e^{r|k|}
$$

where

$$
\left|f_{k}\right|_{s, h}=\sup _{|z| \leq s, p \in \Pi_{h}}\left|f_{k}(z ; p)\right|
$$

And, we can do the same procedure for the function $g(x, z ; p)$.

Now we are in a position to state our main result.

Theorem 2.7. Consider the real analytic quasi-periodic mapping $\mathfrak{M}$ given by (1.5), which is reversible with respect to the involution $\mathcal{R}:(x, z) \mapsto(-x, z)$, that is, $\mathfrak{M} \mathcal{R} \mathfrak{M}=\mathcal{R}$. We assume that the corresponding shell functions $F(\theta, z ; p), G(\theta, z ; p)$ of $f(x, z ; p), g(x, z ; p)$ are real analytic on $D(r, s) \times \Pi_{h}$ with

$$
\|f\|_{r, s, h}+\|g\|_{r, s, h}=\varepsilon<\frac{\gamma}{50 \Lambda(\tau)}
$$

where $\tau$ is so large such that

$$
16 \int_{\tau}^{\infty} \frac{\ln \Lambda(t)}{t^{2}} d t<\frac{r}{2}
$$

Then there is a non-empty Cantor set $\Pi_{\omega, \gamma, \Delta}^{*} \subseteq \Pi_{\omega, \gamma, \Delta}$ and a family of transformations $\mathfrak{V}_{*}(\cdot, \cdot ; p): D_{*} \rightarrow D(r, s)$ for all $p \in \Pi_{\omega, \gamma, \Delta}^{*}$,

$$
x=\xi+u_{*}(\xi ; p), \quad z=v_{*}(\xi ; p)
$$

satisfying

$$
\left\|\mathfrak{V}_{*}-\mathrm{id}\right\|_{D_{*} \times \Pi_{\omega, \gamma, \Delta}^{*}} \leq \exp \left\{\frac{4 d a \Lambda_{0} \varepsilon_{0}}{(d-1) \gamma}\right\} \frac{2 d \Lambda_{0} \varepsilon_{0} \sigma}{(d-1) \gamma}
$$


where $D_{*}=\left\{|\operatorname{Im} \xi|<r_{*}\right\} \times\{0\}, r / 2 \leq r_{*}<r$. Under these transformations, the mapping $\mathfrak{M}$ is transformed into

$$
\mathfrak{M}_{*}: \quad \xi_{1}=\xi+\Omega_{*}(p), \quad \eta_{1}=\eta
$$

Thus, for any $p \in \Pi_{\omega, \gamma, \Delta}^{*}$ the mapping (1.5) has an invariant curve $\Gamma_{0}$ with the form

$$
x=x^{\prime}+\varphi\left(x^{\prime}\right), \quad z=\psi\left(x^{\prime}\right),
$$

where $\varphi, \psi$ are real analytic quasi-periodic with the frequency $\omega=\left(\omega_{1}, \omega_{2}, \ldots, \omega_{m}\right)$, and the invariant curve $\Gamma_{0}$ is of the form $z=\phi(x)$ with $\phi \in Q_{r_{*}}(\omega)$. The restriction of $\mathfrak{M}$ onto $\Gamma_{0}$ is

$$
\left.\mathfrak{M}\right|_{\Gamma_{0}}: \quad x_{1}^{\prime}=x^{\prime}+\Omega_{*}(p)
$$

whose the frequency satisfies that

$$
\left|\Omega_{*}(p)-\Omega(p)\right|_{\Pi_{\omega, \gamma, \Delta}^{*}} \leq 2 \varepsilon
$$

and

$$
\left|\langle k, \omega\rangle \frac{\Omega_{*}(p)}{2 \pi}-j\right| \geq \frac{\gamma}{2 \Delta(|k|)} \quad \text { for all } k \in \mathbb{Z}^{m} \backslash\{0\}, j \in \mathbb{Z} .
$$

Moreover, we have meas $\left(\Pi_{\omega, \gamma, \Delta} \backslash \Pi_{\omega, \gamma, \Delta}^{*}\right) \leq c \gamma^{1 / \lambda}$.

\section{Proof of the main result}

In this section we will prove Theorem 2.7. The proof is based on the KAM approach, is to find a sequence of changes of variables such that the transformed mapping of $\mathfrak{M}$ will be closer to

$$
x_{1}=x+\Omega_{*}(p), \quad z_{1}=z
$$

than the previous one in the narrower domain.

In the KAM step, under the weaker non-degeneracy condition 2.2 , the frequencies can not be regarded as independent parameters. Moreover, the frequencies may have some drifts at each step, rather than the frequencies remain unchanged. Hence the previous methods in 17, 18, 21, 34] are not valid. The key is to control the parameters in the small divisors. We use the ideas in 26, 28, 33, in the proof we use a new variant of the KAM theory which containing an artificial parameter $q, 0<q<1$ and makes the steps of the KAM iteration infinitely small in the speed of function $q^{n} \varepsilon$. In the following, we first give one KAM step, we will give a construction of such transformation. 


\subsection{The KAM step}

Lemma 3.1. Consider the following real analytic quasi-periodic reversible mappings

$$
\mathfrak{M}:\left\{\begin{array}{l}
x_{1}=x+\Omega(p)+f(x, z ; p) \\
z_{1}=z+g(x, z ; p)
\end{array}\right.
$$

on $D(r, s) \times \Pi_{h}$. Let $0<\sigma<r / 2, \tau \geq 1$, define $a=1-e^{-\tau \sigma}$. For all $p \in \Pi_{\omega, \gamma, \Delta}$,

$$
\left|\langle k, \omega\rangle \frac{\Omega(p)}{2 \pi}-j\right| \geq \frac{\gamma}{\Delta(|k|)} \quad \text { for all } k \in \mathbb{Z}^{m} \backslash\{0\}, j \in \mathbb{Z}, 0<|k| \leq \tau .
$$

Let

$$
h=\frac{\pi \gamma}{\Lambda(\tau) T|\omega|_{\infty}}, \quad \max _{p \in \Pi_{h}}\left|\Omega^{\prime}(p)\right| \leq T .
$$

Suppose that

$$
\|f\|_{r, s, h}+\|g\|_{r, s, h} \leq \varepsilon<\frac{b \gamma}{2 \Lambda(\tau)}
$$

where $0<b \leq 1 / 2$ is a positive constant. Then for any $p \in \Pi_{h}$, there exists a change of variables $\mathfrak{U}$

$$
x=\xi+u(\xi, \eta ; p), \quad z=\eta+v(\xi, \eta ; p),
$$

where $u$ and $v$ are real analytic quasi-periodic in $\xi$ with the frequency $\omega=\left(\omega_{1}, \omega_{2}, \ldots, \omega_{m}\right)$. Under this transformation, the original mapping $\mathfrak{M}$ is changed into the form

$$
\mathfrak{M}_{+}=\mathfrak{U}^{-1} \mathfrak{M} \mathfrak{U}:\left\{\begin{array}{l}
\xi_{1}=\xi+\Omega_{+}(p)+f_{+}(\xi, \eta ; p) \\
\eta_{1}=\eta+g_{+}(\xi, \eta ; p)
\end{array}\right.
$$

where the new perturbation $f_{+}$and $g_{+}$are real analytic quasi-periodic functions in $\xi$ with the frequency $\omega=\left(\omega_{1}, \omega_{2}, \ldots, \omega_{m}\right)$ defined in a smaller domain $D\left(r_{+}, s_{+}\right)$and satisfies

$$
\left\|f_{+}\right\|_{r_{+}, s_{+}, h}+\left\|g_{+}\right\|_{r_{+}, s_{+}, h} \leq \varepsilon_{+}=q \epsilon,
$$

where

$$
r_{+}=r-\sigma, \quad 0<s_{+}=s-\frac{\sigma}{a} \leq \varepsilon, \quad q=\frac{2\left(a^{2} b+b+a b+(1-a)+\frac{b}{2 a}\right)}{1-2 a b},
$$

$a, b$ are positive constants such that $0<q<1$. And,

$$
\left|\Omega_{+}(p)-\Omega(p)\right| \leq a \varepsilon \quad \text { for all } p \in \Pi_{h} .
$$

Let $\gamma_{+}=\gamma-\frac{a \Lambda(\tau)|\omega|_{\infty} \varepsilon}{2 \pi}$, define

$$
\mathrm{R}_{\omega, \gamma, \Delta}^{+}=\bigcup_{\substack{\tau<|k| \leq \tau_{+} \\ k \in \mathbb{Z}^{m} \backslash\{0\}, j \in \mathbb{Z}}} \mathrm{R}_{\omega, \gamma, \Delta}^{+, k, j}=\bigcup_{\substack{\tau<|k| \leq \tau_{+} \\ k \in \mathbb{Z}^{m} \backslash\{0\}, j \in \mathbb{Z}}}\left\{p \in \Pi_{\omega, \gamma}:\left|\langle k, \omega\rangle \frac{\Omega_{+}(p)}{2 \pi}-j\right|<\frac{\gamma_{+}}{\Delta(|k|)}\right\},
$$


$\Pi_{\omega, \gamma, \Delta}^{+}=\Pi_{\omega, \gamma, \Delta} \backslash \mathrm{R}_{\omega, \gamma, \Delta}^{+}$. Then for all $p \in \Pi_{\omega, \gamma, \Delta}^{+}$,

$$
\left|\langle k, \omega\rangle \frac{\Omega_{+}(p)}{2 \pi}-j\right| \geq \frac{\gamma_{+}}{\Delta(|k|)} \quad \text { for all } k \in \mathbb{Z}^{m} \backslash\{0\}, j \in \mathbb{Z}, 0<|k| \leq \tau_{+},
$$

where

$$
\Lambda_{+}=\frac{\Lambda(\tau)}{d q}, \quad \tau_{+}=\Lambda^{-1}\left(\Lambda_{+}\right)
$$

Let

$$
T_{+}=T+\frac{a \varepsilon}{(1-d q) h}, \quad h_{+}=\frac{\pi \gamma_{+}}{\Lambda\left(\tau_{+}\right) T_{+}|\omega|_{\infty}},
$$

where $d>1$ is a constant such that $0<d q<1$. If $h_{+} \leq d q$, then $\max _{p \in \Pi_{h_{+}}}\left|\Omega_{+}^{\prime}(p)\right| \leq T_{+}$. Moreover,

$$
\left\|f_{+}\right\|_{r_{+}, s_{+}, h_{+}}+\left\|g_{+}\right\|_{r_{+}, s_{+}, h_{+}} \leq \varepsilon_{+}=q \epsilon .
$$

Thus the above result also holds for the new mapping $\mathfrak{M}_{+}$in place of the mapping $\mathfrak{M}$.

Proof. Lemma 3.1 is usually called the iteration lemma which is actually one KAM step. We divide the KAM step into several small steps.

\subsubsection{Truncation}

The function $f(x, z ; p)$ is real analytic quasi-periodic in $x$ with the frequency $\omega=\left(\omega_{1}, \omega_{2}\right.$, $\left.\ldots, \omega_{m}\right)$, expand $f(x, z ; p)$ as Fourier series with respect to $x$,

$$
f(x, z ; p)=\sum_{k \in \mathbb{Z}^{m}} f_{k}(z ; p) e^{i\langle k, \omega\rangle x}
$$

Then we truncate $f(x, z ; p)=\widetilde{f}(x, z ; p)+\widehat{f}(x, z ; p)$ with

$$
\widehat{f}(x, z ; p)=\sum_{\substack{k \in \mathbb{Z}^{m} \\|k|>\tau}} f_{k}(z ; p) e^{i\langle k, \omega\rangle x}+(1-a) \sum_{\substack{k \in \mathbb{Z}^{m} \\|k| \leq \tau}} f_{k}(z ; p) e^{|k| \sigma} e^{i\langle k, \omega\rangle x},
$$

and

$$
\widetilde{f}(x, z ; p)=\sum_{\substack{k \in \mathbb{Z}^{m} \\|k| \leq \tau}} \widetilde{f}_{k}(z ; p) e^{i\langle k, \omega\rangle x}, \quad \widetilde{f}_{k}(z ; p)=\left(1-(1-a) e^{|k| \sigma}\right) f_{k}(z ; p) .
$$

In view of $e^{-\tau \sigma}=1-a$, we have

$$
\|\widehat{f}\|_{r-\sigma, s, h} \leq(1-a)\|f\|_{r, s, h} \leq(1-a) \varepsilon .
$$

On the other hand, the rest $\widetilde{f}(x, z ; p)$ is bounded. Indeed, with $\widetilde{\sigma}=\frac{\sigma(1-a)}{a}$,

$$
\begin{aligned}
\|\widetilde{f}\|_{r+\widetilde{\sigma}, s, h} & =\sum_{\substack{k \in \mathbb{Z}^{m} \\
0 \leq|k| \leq \tau}}\left(1-(1-a) e^{|k| \sigma}\right)\left|f_{k}\right|_{s, h} e^{|k|(r+\widetilde{\sigma})} \\
& \leq \sup _{0 \leq t \leq \tau}\left(1-(1-a) e^{t \sigma}\right) e^{t \widetilde{\sigma}} \sum_{\substack{k \in \mathbb{Z}^{m} \\
0 \leq|k| \leq \tau}}\left|f_{k}\right|_{s, h} e^{|k| r}=a \varepsilon
\end{aligned}
$$


which is guaranteed by the function $\sup _{0 \leq t \leq \tau}\left(1-(1-a) e^{t \sigma}\right) e^{t \widetilde{\sigma}}$ which is monotonically decreasing for $0 \leq t \leq \tau$ and equals $a$ at $t=0$.

Similarly, $g(x, z ; p)$ has the same decomposition and estimates.

\subsubsection{Extension of small divisors estimate}

For any $p \in \Pi_{h}$, there is a parameter $p_{0} \in \Pi_{\omega, \gamma, \Delta}$ with $\left|p-p_{0}\right|<h=\frac{\pi \gamma}{\Lambda(\tau) T|\omega|_{\infty}}$, hence in view of $\Lambda(t)=t \Delta(t), k \in \mathbb{Z}^{m}, 0<|k| \leq \tau$,

$$
\left|\langle k, \omega\rangle\left(\Omega(p)-\Omega\left(p_{0}\right)\right)\right| \leq|k||\omega|_{\infty}\left|\Omega(p)-\Omega\left(p_{0}\right)\right| \leq \tau|\omega|_{\infty} T h=\frac{\pi \gamma}{\Delta(\tau)} .
$$

As $p_{0} \in \Pi_{\omega, \gamma, \Delta}$, then $\Omega\left(p_{0}\right)$ satisfies (3.1), all relevant divisors thus admit the lower bound

$$
\begin{aligned}
\left|\langle k, \omega\rangle \frac{\Omega(p)}{2 \pi}-j\right| & \geq\left|\langle k, \omega\rangle \frac{\Omega\left(p_{0}\right)}{2 \pi}-j\right|-\left|\langle k, \omega\rangle \frac{\Omega(p)-\Omega\left(p_{0}\right)}{2 \pi}\right| \\
& \geq \frac{\gamma}{\Delta(|k|)}-\frac{\gamma}{2 \Delta(\tau)} \geq \frac{\gamma}{2 \Delta(|k|)} .
\end{aligned}
$$

Thus the non-resonant condition (3.1) also holds for all $p$ in the neighborhood $\Pi_{h}$ of $\Pi_{\omega, \gamma, \Delta}$ with $\gamma=\gamma / 2$.

\subsubsection{Construction of the transformation}

From the theory of transformations, we know that after a canonical change of variables, the transformed mapping of a symplectic map is also symplectic. Analogously, for a reversible mapping, it is easy to see that if the change of variables commutes with the involution $\mathcal{R}$, then the transformed mapping is also reversible with respect to the same involution $\mathcal{R}$. More precisely, if the change of variables $(\xi, \eta) \mapsto(x, z)$ is of the form

$$
\mathfrak{U}:\left\{\begin{array}{l}
x=\xi+u(\xi, \eta ; p), \\
z=\eta+v(\xi, \eta ; p),
\end{array}\right.
$$

then from the equality $\mathcal{R} \mathfrak{U}=\mathfrak{U} \mathcal{R}$, it follows that

$$
u(-\xi, \eta ; p)=-u(\xi, \eta ; p), \quad v(-\xi, \eta ; p)=v(\xi, \eta ; p)
$$

In this case, the transformed mapping $\mathfrak{U}^{-1} \mathfrak{M} \mathfrak{U}$ of $\mathfrak{M}$ is also reversible with respect to the involution $\mathcal{R}:(\xi, \eta) \mapsto(-\xi, \eta)$.

In the construction of the transformation, we will meet the following difference equation (the so-called homological equation):

$$
l(x+\alpha)-l(x)=\mathbf{h}(x),
$$

where $\mathbf{h}=\sum_{\substack{k \in \mathbb{Z}^{m} \\|k| \leq \tau}} h_{k} e^{i\langle k, \omega\rangle x} \in Q_{r}(\omega)$. Let us first study this equation. 
Lemma 3.2. Suppose that $\mathbf{h} \in Q_{r}(\omega)$ and $\omega=\left(\omega_{1}, \ldots, \omega_{m}\right)$, $\alpha$ satisfy the BrjunoRüssmann's non-resonant condition

$$
\left|\langle k, \omega\rangle \frac{\alpha}{2 \pi}-j\right| \geq \frac{\gamma}{\Delta(|k|)} \quad \text { for all } k \in \mathbb{Z}^{m} \backslash\{0\}, j \in \mathbb{Z}, 0<|k| \leq \tau .
$$

Then the difference equation (3.6) has the unique solution $l \in Q_{r}(\omega)$ with $\lim _{T \rightarrow \infty} \frac{1}{T} \int_{0}^{T} l(x)$ $d x=0$ if and only if

$$
\lim _{T \rightarrow \infty} \frac{1}{T} \int_{0}^{T} \mathbf{h}(x) d x=0 .
$$

In this case, we have the following estimate

$$
\|l\|_{r} \leq \gamma^{-1} \Delta(\tau)\|\mathbf{h}\|_{r} .
$$

Moreover, if $\mathbf{h}(-x-\alpha)=\mathbf{h}(x)$, then $l$ is odd in $x$; if $\mathbf{h}(-x-\alpha)=-\mathbf{h}(x)$, then $l$ is even in $x$.

Proof. From $\mathbf{h} \in Q_{r}(\omega)$ and (3.7), (3.8), we know that $\mathbf{h}$ can be represented by

$$
\mathbf{h}(x)=\sum_{\substack{k \in \mathbb{Z}^{m} \\ 0<|k| \leq \tau}} \mathbf{h}_{k} e^{i\langle k, \omega\rangle x}
$$

If using a Fourier series representation

$$
l(x)=\sum_{k \in \mathbb{Z}^{m}} l_{k} e^{i\langle k, \omega\rangle x} .
$$

After straightforward calculations we obtain the relation between Fourier coefficients $\mathbf{h}_{k}$ and $l_{k}$ as follows

$$
l_{k}=\frac{\mathbf{h}_{k}}{e^{i\langle k, \omega\rangle \alpha}-1}, \quad k \in \mathbb{Z}^{m} \backslash\{0\},|k| \leq \tau,
$$

then $l$ is of the form

$$
l(x)=\sum_{\substack{k \in \mathbb{Z}^{m} \\ 0<|k| \leq \tau}} \frac{\mathbf{h}_{k}}{e^{i\langle k, \omega\rangle \alpha}-1} e^{i\langle k, \omega\rangle x},
$$

which is the uniquely determined Fourier expansion of the wanted solution $l$ satisfying $l \in Q(\omega)$ with $\lim _{T \rightarrow \infty} \frac{1}{T} \int_{0}^{T} l(x) d x=0$.

From (3.7), it follows that

$$
\left|e^{i\langle k, \omega\rangle \alpha}-1\right| \geq\left|\langle k, \omega\rangle \frac{\alpha}{2 \pi}-j\right| \geq \frac{\gamma}{\Delta(|k|)} \quad \text { for all } k \in \mathbb{Z}^{m} \backslash\{0\}, 0<|k| \leq \tau .
$$

Hence

$$
\|l\|_{r} \leq \sum_{\substack{k \in \mathbb{Z}^{m} \\ 0<|k| \leq \tau}} \gamma^{-1} \Delta(|k|)\left|\mathbf{h}_{k}\right| e^{|k| r} \leq \gamma^{-1} \Delta(\tau)\|\mathbf{h}\|_{r}
$$


When $\mathbf{h}(-x-\alpha)=\mathbf{h}(x)$, we have

$$
\mathbf{h}_{k}=\mathbf{h}_{-k} e^{i\langle k, \omega\rangle \alpha}
$$

which yields that

$$
\begin{aligned}
l(-x) & =\sum_{\substack{k \in \mathbb{Z}^{m} \\
0<|k| \leq \tau}} \frac{\mathbf{h}_{k}}{e^{i\langle k, \omega\rangle \alpha}-1} e^{-i\langle k, \omega\rangle x}=\sum_{\substack{-k \in \mathbb{Z}^{m} \\
0<|k| \leq \tau}} \frac{\mathbf{h}_{-k}}{e^{-i\langle k, \omega\rangle \alpha-1}} e^{i\langle k, \omega\rangle x} \\
& =-\sum_{\substack{k \in \mathbb{Z}^{m} \\
0<|k| \leq \tau}} \frac{\mathbf{h}_{k}}{e^{i\langle k, \omega\rangle \alpha}-1} e^{i\langle k, \omega\rangle x}=-l(x) .
\end{aligned}
$$

Similarly, one may verify that $l(-x)=l(x)$ if $\mathbf{h}(-x-\alpha)=-\mathbf{h}(x)$. The proof of this lemma is completed.

We shall construct a change of variables $\mathfrak{U}$ :

$$
x=\xi+u(\xi, \eta ; p), \quad z=\eta+v(\xi, \eta ; p),
$$

where $u$ and $v$ are real analytic quasi-periodic in $\xi$ with the frequency $\omega=\left(\omega_{1}, \omega_{2}, \ldots, \omega_{m}\right)$. Under this transformation, the original mapping $\mathfrak{M}$ is changed into the form

$$
\mathfrak{M}_{+}=\mathfrak{U}^{-1} \mathfrak{M} \mathfrak{U}: \quad\left\{\begin{array}{l}
\xi_{1}=\xi+\Omega_{+}(p)+f_{+}(\xi, \eta ; p) \\
\eta_{1}=\eta+g_{+}(\xi, \eta ; p)
\end{array}\right.
$$

where the functions $f_{+}$and $g_{+}$are real analytic quasi-periodic functions in $\xi$ with the frequency $\omega=\left(\omega_{1}, \omega_{2}, \ldots, \omega_{m}\right)$ defined in a smaller domain $D\left(s_{+}, r_{+}\right) \times \Pi_{h_{+}}$and $\left\|f_{+}\right\|_{r_{+}, s_{+}, h_{+}}$ $+\left\|g_{+}\right\|_{r_{+}, s_{+}, h_{+}} \leq \varepsilon_{+}=q \epsilon$ is smaller than $\|f\|_{r, s, h}+\|g\|_{r, s, h}$.

We also assume that for each fixed $y, p, f(\cdot, y ; p), g(\cdot, y ; p) \in Q_{r}(\omega)$ and $f, g$ are real analytic in the domain $D(r, s) \times \Pi_{h}$. Moreover, we assume that $\omega=\left(\omega_{1}, \ldots, \omega_{m}\right), \Omega(p)$ satisfy the non-resonance conditions 2.1 and $(2.3)$. Let

$$
\|f\|_{r, s, h}+\|g\|_{r, s, h} \leq \varepsilon
$$

We try to motivate the following constructions for $u, v$ first. From (3.10) and (3.11), it follows that

$$
\begin{aligned}
& f_{+}(\xi, \eta ; p)=f(\xi+u, \eta+v ; p)+u(\xi, \eta ; p)-u\left(\xi_{1}, \eta_{1} ; p\right)+\Omega(p)-\Omega_{+}(p) \\
& g_{+}(\xi, \eta ; p)=g(\xi+u, \eta+v ; p)+v(\xi, \eta ; p)-v\left(\xi_{1}, \eta_{1} ; p\right)
\end{aligned}
$$

which serve to define $f_{+}, g_{+}$implicity in $D\left(r_{+}, s_{+}\right) \times \Pi_{h_{+}}$. If $u(\cdot, \eta ; p), v(\cdot, \eta ; p) \in Q(\omega)$, then one can prove that $f_{+}(\cdot, \eta ; p), g_{+}(\cdot, \eta ; p) \in Q(\omega)$ are well defined by $(3.12)$. Indeed, since $f(\cdot, z ; p), g(\cdot, z ; p), u(\cdot, \eta ; p), v(\cdot, \eta ; p) \in Q(\omega)$, then we have $f(\xi+u, \eta+v ; p), g(\xi+$ 
$u, \eta+v ; p) \in Q(\omega)$. Hence $f(\xi+u, \eta+v ; p)+u(\xi, \eta ; p), g(\xi+u, \eta+v ; p)+v(\xi, \eta ; p) \in$ $Q(\omega)$. Denote $\widetilde{\phi}(\xi, \eta ; p)=f(\xi+u, \eta+v ; p)+u(\xi, \eta ; p)+\Omega(p)-\Omega_{+}(p), \widetilde{\psi}(\xi, \eta ; p)=$ $g(\xi+u, \eta+v ; p)+v(\xi, \eta ; p)$, and the corresponding shell functions are $\widetilde{\Phi}(\theta, \eta ; p), \widetilde{\Psi}(\theta, \eta ; p)$ which are period $2 \pi$ in each of the variables $\theta_{i}(1 \leq i \leq m) . U(\theta, \eta ; p), V(\theta, \eta ; p)$ are the corresponding shell functions of $u(\xi, \eta ; p), v(\xi, \eta ; p)$. If the unknown functions $f_{+}(\xi, \eta ; p)$, $g_{+}(\xi, \eta ; p)$ are represented by $F_{+}(\theta, \eta ; p), G_{+}(\theta, \eta ; p)$, where $f_{+}(\xi, \eta ; p)=F_{+}(\omega \xi, \eta ; p)$, $g_{+}(\xi, \eta ; p)=G_{+}(\omega \xi, \eta ; p)$, the conditions for $F_{+}, G_{+}$become

$$
\begin{aligned}
F_{+}(\theta, \eta ; p) & =\widetilde{\Phi}(\theta, \eta ; p)-U\left(\theta+\omega \eta+\omega F_{+}(\theta, \eta ; p), \eta+G_{+}(\theta, \eta ; p) ; p\right), \\
\left.G_{+}(\theta, \eta ; p)\right) & =\widetilde{\Psi}(\theta, \eta ; p)-V\left(\theta+\omega \eta+\omega F_{+}(\theta, \eta ; p), \eta+G_{+}(\theta, \eta ; p) ; p\right) .
\end{aligned}
$$

By (3.17), (3.24) and the implicit function theorem, we know that $F_{+}(\theta, \eta ; p), G_{+}(\theta, \eta ; p)$ are well defined by (3.13), and have period $2 \pi$ in each of the variables $\theta_{i}(1 \leq i \leq m)$.

In the following, we will determine the unknown functions $u$ and $v$ to satisfy the condition (3.5) in order to guarantee that the transformed mapping $\mathfrak{M}_{+}=\mathfrak{U}^{-1} \mathfrak{M} \mathfrak{U}$ is also reversible.

As one did in the periodic case, we may solve $u$ and $v$ from the following equations

$$
\begin{aligned}
& u(\xi+\Omega(p), \eta ; p)-u(\xi, \eta ; p)=\widetilde{f}(\xi, \eta ; p)-[\widetilde{f}](\eta ; p), \\
& v(\xi+\Omega(p), \eta ; p)-v(\xi, \eta ; p)=\widetilde{g}(\xi, \eta ; p)-[\widetilde{g}](\eta ; p)
\end{aligned}
$$

where $[\cdot]$ denotes the mean value of a function over the first variable. Indeed, one can solve these functions from the above equation. But the problem is that such functions $u$ and $v$ do not, in general, satisfy the condition (3.5) i.e., the transformation $\mathfrak{U}$ does not commute with the involution $\mathcal{R}$. In this case, the transformed mapping $\mathfrak{M}_{+}=\mathfrak{U}^{-1} \mathfrak{M} \mathfrak{U}$ is no longer a reversible mapping with respect to $\mathcal{R}$. Therefore, we cannot use the above equations to determine the functions $u$ and $v$. Instead of solving the above equations, we may find these functions $u$ and $v$ from the following modified homological equations

$$
\begin{aligned}
& u(\xi+\Omega(p), \eta ; p)-u(\xi, \eta ; p)=\widetilde{F}(\xi, \eta ; p), \\
& v(\xi+\Omega(p), \eta ; p)-v(\xi, \eta ; p)=\widetilde{G}(\xi, \eta ; p),
\end{aligned}
$$

where

$$
\begin{aligned}
\widetilde{F}(\xi, \eta ; p) & =\frac{1}{2}(\widetilde{f}(\xi, \eta ; p)+\widetilde{f}(-\xi-\Omega(p), \eta ; p))-[\widetilde{f}](\eta ; p), \\
\widetilde{G}(\xi, \eta ; p) & =\frac{1}{2}(\widetilde{g}(\xi, \eta ; p)-\widetilde{g}(-\xi-\Omega(p), \eta ; p)) .
\end{aligned}
$$

It is easy to verify that $\widetilde{F}(-\xi-\Omega(p), \eta ; p)=\widetilde{F}(\xi, \eta ; p)$ and $\widetilde{G}(-\xi-\Omega(p), \eta ; p)=-\widetilde{G}(\xi, \eta ; p)$, hence the functions $u$ and $v$ meet the condition 3.5 by Lemma 3.2. In this case, the transformed mapping $\mathfrak{M}_{+}=\mathfrak{U}^{-1} \mathfrak{M} \mathfrak{U}$ is also reversible with respect to the involution $\mathcal{R}:(\xi, \eta) \mapsto(-\xi, \eta)$. 
Now we can solve the functions $u$ and $v$ from (3.14) and give the estimates of them. From the Lemma 3.2 , we know $u$ and $v$ have a Fourier series expansion

$$
u=\sum_{k \in \mathbb{Z}^{m}} u_{k} e^{i\langle k, \omega\rangle x}, \quad v=\sum_{k \in \mathbb{Z}^{m}} v_{k} e^{i\langle k, \omega\rangle x},
$$

where

$$
u_{k}=\frac{\tilde{f}_{k}}{e^{i\langle k, \omega\rangle \Omega(p)}-1}, \quad v_{k}=\frac{\widetilde{g}_{k}}{e^{i\langle k, \omega\rangle \Omega(p)}-1}, \quad k \in \mathbb{Z}^{m} \backslash\{0\},|k| \leq \tau .
$$

By (3.4), 3.9 and $a=1-e^{-\tau \sigma}<\tau \sigma$ we get

$$
\|u\|_{r+\widetilde{\sigma}, s, h} \leq 2 \Delta(\tau) \gamma^{-1}\|\tilde{f}\|_{r+\widetilde{\sigma}, s, h} \leq 2 \Delta(\tau) \gamma^{-1} a \varepsilon \leq 2 \Lambda(\tau) \gamma^{-1} \sigma \varepsilon
$$

Similarly,

$$
\|v\|_{r+\widetilde{\sigma}, s, h} \leq 2 \Lambda(\tau) \gamma^{-1} \sigma \varepsilon
$$

Because

$$
r+\widetilde{\sigma}-(r-\sigma)=\frac{(1-a) \sigma}{a}+\sigma=\frac{\sigma}{a}
$$

by Cauchy's estimates and (3.15), 3.16), we have

$$
\begin{aligned}
\left\|u_{\xi}\right\|_{r-\sigma, s, h} & \leq \frac{a}{\sigma}\|u\|_{r+\widetilde{\sigma}, s, h} \leq 2 a \Lambda(\tau) \gamma^{-1} \varepsilon, \\
\left\|v_{\xi}\right\|_{r-\sigma, s, h} & \leq \frac{a}{\sigma}\|v\|_{r+\widetilde{\sigma}, s, h} \leq 2 a \Lambda(\tau) \gamma^{-1} \varepsilon, \\
\left\|u_{\eta}\right\|_{r+\widetilde{\sigma}, s-\sigma / a, h} & \leq \frac{a}{\sigma}\|u\|_{r+\widetilde{\sigma}, s, h} \leq 2 a \Lambda(\tau) \gamma^{-1} \varepsilon, \\
\left\|v_{\eta}\right\|_{r+\widetilde{\sigma}, s-\sigma / a, h} & \leq \frac{a}{\sigma}\|u\|_{r+\widetilde{\sigma}, s, h} \leq 2 a \Lambda(\tau) \gamma^{-1} \varepsilon .
\end{aligned}
$$

\subsubsection{Estimates of the new frequency}

However, due to the lack of twist condition, we have $[\widetilde{f}](\eta ; p)$ in every KAM step, which will be an obstruction in making the new perturbation smaller enough to go to the next iteration. Therefore, the frequencies can not be regarded as independent parameters. Moreover, the frequencies may have some drifts at each step, we have to introduce

$$
\Omega_{+}(p)=\Omega(p)+[\widetilde{f}](0 ; p)
$$

as new frequencies, where $[\widetilde{f}](0 ; p)$ denotes the value of $[\widetilde{f}](\eta ; p)$ at $\eta=0$. As a consequence, we have

$$
\left|\Omega_{+}(p)-\Omega(p)\right| \leq\|\widetilde{f}\|_{r+\widetilde{\sigma}, s, h} \leq a \varepsilon \quad \text { for all } p \in \Pi_{h}
$$


it follows that 3.2 holds. Let $\gamma_{+}=\gamma-\frac{a \Lambda(\tau)|\omega|_{\infty} \varepsilon}{2 \pi}$. We have

$$
\begin{aligned}
\left|\langle k, \omega\rangle \frac{\Omega_{+}(p)}{2 \pi}-j\right| & \geq\left|\langle k, \omega\rangle \frac{\Omega(p)}{2 \pi}-j\right|-\left|\langle k, \omega\rangle \frac{\Omega_{+}(p)-\Omega(p)}{2 \pi}\right| \geq \frac{\gamma}{\Delta(|k|)}-\frac{\tau|\omega|_{\infty} a \varepsilon}{2 \pi} \\
& \geq \frac{1}{\Delta(|k|)}\left(\gamma-\frac{a \tau \Delta(\tau)|\omega|_{\infty} \varepsilon}{2 \pi}\right)=\frac{\gamma_{+}}{\Delta(|k|)}
\end{aligned}
$$

for all $p \in \Pi_{\omega, \gamma, \Delta}, k \in \mathbb{Z}^{m} \backslash\{0\}, j \in \mathbb{Z}, 0<|k| \leq \tau$, that is, the non-resonant condition with respect to the new frequency $\Omega_{+}(p)$ automatically holds for $k \in \mathbb{Z}^{m} \backslash\{0\}, j \in \mathbb{Z}$, $0<|k| \leq \tau$. Then by the definition of $\mathrm{R}_{\omega, \gamma, \Delta}^{+}$, it follows that 3.3 holds.

\subsubsection{Estimates of the new perturbation}

From 3.12, 3.14) and (3.18), we have

$$
\begin{aligned}
f_{+}= & u(\xi+\Omega(p), \eta ; p)-u\left(\xi_{1}, \eta_{1} ; p\right)+f(\xi+u, \eta+v ; p) \\
- & \frac{1}{2}(\widetilde{f}(\xi, \eta ; p)+\widetilde{f}(-\xi-\Omega(p), \eta ; p))+[\widetilde{f}](\eta ; p)-[\widetilde{f}](0 ; p), \\
g_{+}= & v(\xi+\Omega(p), \eta ; p)-v\left(\xi_{1}, \eta_{1} ; p\right)+g(\xi+u, \eta+v ; p) \\
& -\frac{1}{2}(\widetilde{g}(\xi, \eta ; p)-\widetilde{g}(-\xi-\Omega(p), \eta ; p)) .
\end{aligned}
$$

From the reversibility of $\mathfrak{M}$, it follows that

$$
\begin{gathered}
f(-x-\Omega(p)-f, z+g ; p)-f(x, z ; p)=0 \\
g(-x-\Omega(p)-f, z+g ; p)+g(x, z ; p)=0 .
\end{gathered}
$$

By 3.19-3.21, we obtain

$$
\begin{aligned}
f_{+}= & u(\xi+\Omega(p), \eta ; p)-u\left(\xi_{1}, \eta_{1} ; p\right)+f(\xi+u, \eta+v ; p)-f \\
+ & \frac{1}{2}(f-\widetilde{f}+f(-\xi-\Omega(p), \eta ; p)-\widetilde{f}(-\xi-\Omega(p), \eta ; p) \\
& \quad+f(-\xi-\Omega(p)-f, \eta+g ; p)-f(-\xi-\Omega(p), \eta ; p)) \\
& +[\widetilde{f}](\eta ; p)-[\widetilde{f}](0 ; p)
\end{aligned}
$$

and

$$
\begin{aligned}
g_{+}= & v(\xi+\Omega(p), \eta ; p)-v\left(\xi_{1}, \eta_{1} ; p\right)+g(\xi+u, \eta+v ; p)-g \\
+ & \frac{1}{2}(g-\widetilde{g}-g(-\xi-\Omega(p), \eta ; p)+\widetilde{g}(-\xi-\Omega(p), \eta ; p) \\
& -g(-\xi-\Omega(p)-f, \eta+g ; p)+g(-\xi-\Omega(p), \eta ; p)) .
\end{aligned}
$$


The contribution from the functions $u, v$ on the right-hand side of $(3.22),(3.23)$ can be estimated using the mean value theorem followed by (3.17), yielding

$$
\begin{aligned}
& \left\|u(\xi+\Omega(p), \eta ; p)-u\left(\xi_{1}, \eta_{1} ; p\right)\right\|_{r-\sigma, s-\sigma / a, h} \\
\leq & \left\|u_{\xi}\right\|_{r-\sigma, s, h}\left(\left|\Omega_{+}(p)-\Omega(p)\right|+\left\|f_{+}\right\|_{r-\sigma, s-\sigma / a, h}\right)+\left\|u_{\eta}\right\|_{r-\sigma, s, h}\left\|g_{+}\right\|_{r-\sigma, s-\sigma / a, h} \\
\leq & a^{2} b \varepsilon+a b\left(\left\|f_{+}\right\|_{r-\sigma, s-\sigma / a, h}+\left\|g_{+}\right\|_{r-\sigma, s-\sigma / a, h}\right),
\end{aligned}
$$

where

$$
a=1-e^{-\tau \sigma}, \quad b=2 \Lambda(\tau) \gamma^{-1} \varepsilon,
$$

$0<a<1,0<b \leq 1 / 2$ are positive constants.

Recalling that $\|f\|_{r, s, h}+\|g\|_{r, s, h} \leq \varepsilon$, we can use Cauchy's estimate to the derivatives of $f, g$, we get $\left\|f_{\xi}\right\|_{r-\sigma, s, h},\left\|g_{\xi}\right\|_{r-\sigma, s, h} \leq \varepsilon / \sigma,\left\|f_{\eta}\right\|_{r, s-\sigma / a, h},\left\|g_{\eta}\right\|_{r, s-\sigma / a, h} \leq a \varepsilon / \sigma$, again applying the mean value theorem followed by (3.15), (3.16), we obtain

$$
\begin{gathered}
\quad\|f(\xi+u, \eta+v ; p)-f(\xi, \eta ; p)\|_{r-\sigma, s-\sigma / a, h} \\
\leq\left\|f_{\xi}\right\|_{r-\sigma, s, h}\|u\|_{r-\sigma, s-\sigma / a, h}+\left\|f_{\eta}\right\|_{r, s-\sigma / a, h}\|v\|_{r-\sigma, s-\sigma / a, h} \\
\leq b \varepsilon+a b \varepsilon, \\
\| f-\widetilde{f}+f(-\xi-\Omega(p), \eta ; p)-\widetilde{f}(-\xi-\Omega(p), \eta ; p) \\
+f(-x-\Omega(p)-f, z+g ; p)-f(-\xi-\Omega(p), \eta ; p) \|_{r-\sigma, s-\sigma / a, h} \\
\leq\|f-\widetilde{f}\|_{r-\sigma, s-\sigma / a, h}+\|f(-\xi-\Omega(p), \eta ; p)-\widetilde{f}(-\xi-\Omega(p), \eta ; p)\|_{r-\sigma, s-\sigma / a, h} \\
+\|f(-\xi-\Omega(p)-f, \eta+g ; p)-f(-\xi-\Omega(p), \eta ; p)\|_{r-\sigma, s-\sigma / a, h} \\
\leq(1-a) \varepsilon+(1-a) \varepsilon+\frac{a \varepsilon^{2}}{\sigma} \leq 2(1-a) \varepsilon+b \varepsilon .
\end{gathered}
$$

Since $0<s_{+}=s-\sigma / a \leq \varepsilon$, then we get

$$
\|[\widetilde{f}](\eta ; p)-[\widetilde{f}](0 ; p)\|_{s-\sigma / a, h} \leq \frac{a \varepsilon^{2}}{\sigma} \leq b \varepsilon .
$$

Hence the new perturbation $f_{+}$has the following estimates:

$$
\begin{aligned}
\left\|f_{+}\right\|_{r-\sigma, s-\sigma / a, h} \leq & \left(a^{2} b+a b+(1-a)+\frac{5}{2} b\right) \varepsilon \\
& +a b\left(\left\|f_{+}\right\|_{r-\sigma, s-\sigma / a, h}+\left\|g_{+}\right\|_{r-\sigma, s-\sigma / a, h}\right) .
\end{aligned}
$$

Similarly, for $g_{+}$we get

$$
\begin{aligned}
& \left\|v(\xi+\Omega(p), \eta, ; p)-v\left(\xi_{1}, \eta_{1} ; p\right)\right\|_{r-\sigma, s-\sigma / a, h} \\
\leq & \left\|v_{\xi}\right\|_{r-\sigma, s, h}\left(\left|\Omega_{+}(p)-\Omega(p)\right|+\left\|f_{+}\right\|_{r-\sigma, s-\sigma / a, h}\right)+\left\|v_{\eta}\right\|_{r-\sigma, s, h}\left\|g_{+}\right\|_{r-\sigma, s-\sigma / a, h} \\
\leq & a^{2} b \varepsilon+a b\left(\left\|f_{+}\right\|_{r-\sigma, s-\sigma / a, h}+\left\|g_{+}\right\|_{r-\sigma, s-\sigma / a, h}\right),
\end{aligned}
$$




$$
\begin{gathered}
\|g(\xi+u, \eta+v, p)-g(\xi, \eta, p)\|_{r-\sigma, s-\sigma / a, h} \\
\leq\left\|g_{\xi}\right\|_{r-\sigma, s, h}\|u\|_{r-\sigma, s-\sigma / a, h}+\left\|g_{\eta}\right\|_{r, s-\sigma / a, h}\|v\|_{r-\sigma, s-\sigma / a, h} \\
\leq b \varepsilon+a b \varepsilon, \\
\| g-\widetilde{g}-g(-\xi-\Omega(p), \eta ; p)+\widetilde{g}(-\xi-\Omega(p), \eta ; p) \\
-g(-\xi-\Omega(p)-f, \eta+g ; p)+g(-\xi-\Omega(p), \eta ; p) \|_{r-\sigma, s-\sigma / a, h} \\
\leq\|g-\widetilde{g}\|_{r-\sigma, s-\sigma / a, h}+\|g(-\xi-\Omega(p), \eta ; p)-\widetilde{g}(-\xi-\Omega(p), \eta ; p)\|_{r-\sigma, s-\sigma / a, h} \\
+\|g(-x-\Omega(p)-f, z+g ; p)-g(-\xi-\Omega(p), \eta ; p)\|_{r-\sigma, s-\sigma / a, h} \\
\leq(1-a) \varepsilon+(1-a) \varepsilon+\frac{a \varepsilon^{2}}{\sigma} \leq 2(1-a) \varepsilon+b \varepsilon .
\end{gathered}
$$

The new perturbation $g_{+}$has the following estimates

$$
\begin{aligned}
\left\|g_{+}\right\|_{r-\sigma, s-\sigma / a, h} \leq & \left(a^{2} b+a b+(1-a)+\frac{3}{2} b\right) \varepsilon \\
& +a b\left(\left\|f_{+}\right\|_{r-\sigma, s-\sigma / a, h}+\left\|g_{+}\right\|_{r-\sigma, s-\sigma / a, h}\right) .
\end{aligned}
$$

At last, adding the inequalities (3.25) and (3.26), we have

$$
\begin{aligned}
& \left\|f_{+}\right\|_{r-\sigma, s-\sigma / a, h}+\left\|g_{+}\right\|_{r-\sigma, s-\sigma / a, h} \\
\leq & 2\left(a^{2} b+a b+(1-a)+\frac{5}{2} b\right) \varepsilon+2 a b\left(\left\|f_{+}\right\|_{r-\sigma, s-\sigma / a, h}+\left\|g_{+}\right\|_{r-\sigma, s-\sigma / a, h}\right) .
\end{aligned}
$$

Let

$$
q=\frac{2\left(a^{2} b+a b+(1-a)+\frac{5}{2} b\right)}{1-2 a b}
$$

Then

$$
\left\|f_{+}\right\|_{r-\sigma, s-\sigma / a, h}+\left\|g_{+}\right\|_{r-\sigma, s-\sigma / a, h} \leq q \varepsilon .
$$

Suppose $h_{+} \leq d q h, 0<d q<1$. By Cauchy's estimates and 3.2 we have

$$
\left|\Omega_{+}^{\prime}(p)-\Omega^{\prime}(p)\right| \leq \frac{a \varepsilon}{(1-d q) h} \quad \text { for all } p \in \Pi_{h_{+}} .
$$

Let $T_{+}=T+\frac{a \varepsilon}{(1-d q) h}$, then $\max _{p \in \Pi_{h_{+}}}\left|\Omega_{+}^{\prime}(p)\right| \leq T_{+}$. Moreover, we have

$$
\left\|f_{+}\right\|_{r_{+}, s_{+}, h_{+}}+\left\|g_{+}\right\|_{r_{+}, s_{+}, h_{+}} \leq q \varepsilon
$$

where $r_{+}=r-\sigma, s_{+}=s-\sigma / a, h_{+} \leq d q h$. Thus this ends the proof of Lemma 3.1.

\subsection{Setting the parameters and iteration}

In this section we choose some suitable parameters so that the above iteration can go on infinitely. First, we can always choose $0<a<1$ and $0<b \leq 1 / 2$ such that

$$
0<q=\frac{2\left(a^{2} b+a b+(1-a)+\frac{5}{2} b\right)}{1-2 a b}<1
$$


and we we can even make $d q$ as close to 1 as we wish.

It then suffices to choose for $\varepsilon$ and $\Lambda$ geometric sequences with the same base $q$, namely

$$
\varepsilon_{n}=q^{n} \varepsilon_{0}, \quad \Lambda_{n}=\frac{\Lambda_{0}}{(d q)^{n}}
$$

where we assume $\Lambda_{0} \geq \Lambda(1)=\Delta(1)$. Next, let $\tau_{n}=\Lambda^{-1}\left(\Lambda_{n}\right)$, then $\sum_{n \geq 0} \frac{1}{\tau_{n}}$ is convergent. Indeed, let $t=\Lambda^{-1}\left(\Lambda_{0}(d q)^{-\nu}\right)$, we have

$$
\sum_{n \geq 0} \frac{1}{\tau_{n}} \leq \int_{0}^{\infty} \frac{d \nu}{\Lambda^{-1}\left(\Lambda_{0}(d q)^{-\nu}\right)} \leq \frac{1}{\ln (d q)^{-1}} \int_{\tau_{0}}^{\infty} \frac{d \Lambda(t)}{t \Lambda(t)}
$$

Integrating by parts and requiring $\Lambda\left(\tau_{0}\right) \geq(d q)^{-1}$, we get

$$
\sum_{n \geq 0} \frac{1}{\tau_{n}} \leq \frac{1}{\ln (d q)^{-1}} \int_{\tau_{0}}^{\infty} \frac{\ln \Lambda(t)}{t^{2}} d t
$$

Hence, from the definition of the Brjuno-Rüssmann function $\Delta, \Lambda(t)=t \Delta(t)$, we can achieve that $\sum_{n \geq 0} \frac{1}{\tau_{n}}$ is convergent.

Define then other parameters through

$$
\begin{gathered}
\gamma_{n+1}=\gamma_{n}-\frac{a \Lambda\left(\tau_{n}\right)|\omega|_{\infty} \varepsilon_{n}}{2 \pi}, \quad h_{n}=\frac{\pi \gamma_{n}}{\Lambda\left(\tau_{n}\right) T_{n}|\omega|_{\infty}} \\
T_{n+1}=T_{n}+\frac{a \varepsilon_{n}}{(1-d q) h_{n}}, \quad 1-a=e^{-\tau_{n} \sigma_{n}} \\
r_{n+1}=r_{n}-\sigma_{n}, \quad 0<s_{n+1}=s_{n}-\frac{\sigma_{n}}{a} \leq \varepsilon_{n} .
\end{gathered}
$$

As we will see in a moment, $r_{n}$ has a positive limit and $s_{n}$ tend to 0 for $\Lambda_{0}$ sufficiently large.

Let

$$
\begin{aligned}
\Pi_{\omega, \gamma, \Delta}^{n}= & \left\{p \in \Pi_{\omega, \gamma, \Delta}^{n-1}:\left|\langle k, \omega\rangle \frac{\Omega_{n}(p)}{2 \pi}-j\right| \geq \frac{\gamma_{n}}{\Delta(|k|)}\right. \\
& \text { for all } \left.k \in \mathbb{Z}^{m} \backslash\{0\}, j \in \mathbb{Z}, \tau_{n-1}<|k| \leq \tau_{n}\right\}
\end{aligned}
$$

and

$$
\Pi_{h_{n}}=\left\{p \in \mathbb{C}: \operatorname{dist}\left(p, \Pi_{\omega, \gamma, \Delta}^{n}\right) \leq h_{n}\right\} .
$$

Then for any $p \in \Pi_{h_{n}}$, there is a parameter $p_{0} \in \Pi_{\omega, \gamma, \Delta}^{n}$ with $\left|p-p_{0}\right|<h_{n}=\frac{\pi \gamma_{n}}{\Lambda\left(\tau_{n}\right) T_{n}|\omega|_{\infty}}$, hence in view of $\Lambda\left(\tau_{n}\right)=\tau_{n} \Delta\left(\tau_{n}\right), k \in \mathbb{Z}^{m}, \tau_{n-1}<|k| \leq \tau_{n}$,

$$
\left|\langle k, \omega\rangle\left(\Omega_{n}(p)-\Omega_{n}\left(p_{0}\right)\right)\right| \leq|k||\omega|_{\infty}\left|\Omega_{n}(p)-\Omega_{n}\left(p_{0}\right)\right| \leq \tau_{n}|\omega|_{\infty} T_{n} h_{n}=\frac{\pi \gamma_{n}}{\Delta\left(\tau_{n}\right)} .
$$


As $p_{0} \in \Pi_{\omega, \gamma, \Delta}^{n}$, then $\Omega\left(p_{0}\right)$ satisfies (3.28), all relevant divisors thus admit the lower bound

$$
\begin{aligned}
\left|\langle k, \omega\rangle \frac{\Omega_{n}(p)}{2 \pi}-j\right| & \geq\left|\langle k, \omega\rangle \frac{\Omega_{n}\left(p_{0}\right)}{2 \pi}-j\right|-\left|\langle k, \omega\rangle \frac{\Omega_{n}(p)-\Omega_{n}\left(p_{0}\right)}{2 \pi}\right| \\
& \geq \frac{\gamma_{n}}{\Delta(|k|)}-\frac{\gamma_{n}}{2 \Delta\left(\tau_{n}\right)} \geq \frac{\gamma_{n}}{2 \Delta(|k|)} .
\end{aligned}
$$

Thus the non-resonant condition (3.28) also holds for all $p$ in the neighborhood $\Pi_{h_{n}}$ of $\Pi_{\omega, \gamma, \Delta}^{n}$.

Denote $D_{n}=D\left(r_{n}, s_{n}\right)$ for simplicity. By the iteration lemma, there exists a sequence of transformations $\mathfrak{U}_{n}$ :

$$
x=\xi+u_{n}(\xi, \eta ; p), \quad z=\eta+v_{n}(\xi, \eta ; p),
$$

such that for any $p \in \Pi_{h_{n}}, \mathfrak{U}_{n}: D_{n} \times \Pi_{h_{n}} \rightarrow D_{n-1} \times \in \Pi_{h_{n-1}}$, satisfying

$$
\left\|\mathfrak{U}_{n}-\mathrm{id}\right\|_{D_{n} \times \Pi_{h_{n}}} \leq 2 \Lambda\left(\tau_{n}\right) \gamma_{n}^{-1} \sigma_{n} \varepsilon_{n} \quad \text { and } \quad\left\|D \mathfrak{U}_{n}-\operatorname{Id}\right\|_{D_{n} \times \Pi_{h_{n}}} \leq 2 a \Lambda\left(\tau_{n}\right) \gamma_{n}^{-1} \varepsilon_{n} .
$$

Thus the transformation $\mathfrak{V}_{n}=\mathfrak{U}_{0} \circ \mathfrak{U}_{1} \circ \cdots \circ \mathfrak{U}_{n}$ is well defined in $D_{n} \times \Pi_{h_{n}}$ and is seen to take $\mathfrak{M}_{0}$ into

$$
\mathfrak{M}_{n}=\mathfrak{V}_{n}^{-1} \mathfrak{M}_{0} \mathfrak{V}_{n}
$$

More precisely, if we write $\mathfrak{M}_{0}$ as

$$
x_{1}=x+\Omega(p)+f(x, z ; p), \quad z_{1}=z+g(x, z ; p),
$$

and express $\mathfrak{V}_{n}$ in the form

$$
x=\xi+P_{n}(\xi, \eta ; p), \quad z=\eta+Q_{n}(\xi, \eta ; p),
$$

then $\mathfrak{M}_{0}$ is transformed into $\mathfrak{M}_{n}$ :

$$
\xi_{1}=\xi+\Omega_{n}(p)+f_{n}(\xi, \eta ; p), \quad \eta_{1}=\eta+g_{n}(\xi, \eta ; p),
$$

satisfying

$$
\left\|f_{n}\right\|_{D_{n} \times \Pi_{h_{n}}}+\left\|g_{n}\right\|_{D_{n} \times \Pi_{h_{n}}} \leq \varepsilon_{n}=q^{n} \epsilon_{0} \quad \text { and } \quad\left|\Omega_{n+1}(p)-\Omega_{n}(p)\right|_{\Pi_{h_{n}}} \leq a \varepsilon_{n} .
$$

In the following we will verify the assumptions in Lemma 3.1 to ensure that KAM step is valid for all $n \geq 0$.

By $h_{n}=\frac{\pi \gamma_{n}}{\Lambda\left(\tau_{n}\right) T_{n}|\omega|_{\infty}}$, it follows that

$$
\frac{h_{n+1}}{h_{n}}=\frac{\gamma_{n+1}}{\gamma_{n}} \frac{T_{n}}{T_{n+1}} \frac{\Lambda\left(\tau_{n}\right)}{\Lambda\left(\tau_{n+1}\right)} \leq \frac{\Lambda_{n}}{\Lambda_{n+1}}=d q .
$$


Thus we have $h_{n+1} \leq d q h_{n}$. By the definition of $\gamma_{n}$, we have

$$
\begin{aligned}
\gamma_{n+1} & =\gamma_{n}-\frac{a \Lambda\left(\tau_{n}\right)|\omega|_{\infty} \varepsilon_{n}}{2 \pi}=\gamma_{n}-\frac{a \Lambda_{0}|\omega|_{\infty} \varepsilon_{0}}{2 \pi} \frac{1}{d^{n}} \\
& \geq \gamma_{0}-\frac{a \Lambda_{0}|\omega|_{\infty} \varepsilon_{0}}{2 \pi} \sum_{0 \leq n \in \mathbb{Z}} \frac{1}{d^{n}}=\gamma-\frac{d a \Lambda_{0}|\omega|_{\infty} \varepsilon_{0}}{(d-1) \pi} .
\end{aligned}
$$

Since $\varepsilon_{0} \leq \frac{(d-1) \pi \gamma}{2 d a \Lambda_{0}|\omega|_{\infty}}$, we obtain $\gamma / 2 \leq \gamma_{n} \leq \gamma$ for all $n \geq 1$.

Suppose $\max _{p \in \Pi_{h_{n}}}\left|\Omega_{n}^{\prime}(p)\right| \leq T_{n}$. Let $T_{n+1}=T_{n}+\frac{a \varepsilon_{n}}{(1-d q) h_{n}}$, then we have $\max _{p \in \Pi_{h_{n+1}}}$ $\left|\Omega_{n+1}^{\prime}(p)\right| \leq T_{n+1}$. By iteration,

$$
\begin{aligned}
T_{n+1} & =T_{n}+\frac{a \varepsilon_{n}}{(1-d q) h_{n}} \leq T_{n}\left(1+\frac{2 d a \Lambda_{0}|\omega|_{\infty} \varepsilon_{0}}{\pi(1-d q) \gamma} \frac{1}{d^{n}}\right) \\
& \leq T_{0} \prod_{n \geq 0}\left(1+\frac{2 d a \Lambda_{0}|\omega|_{\infty} \varepsilon_{0}}{\pi(1-d q) \gamma} \frac{1}{d^{n}}\right) .
\end{aligned}
$$

By $\varepsilon_{0} \leq \frac{\pi(1-d q) \gamma}{2 d a \Lambda_{0}|\omega|_{\infty}}$, then $T_{0} \leq T_{n} \leq e^{d /(d-1)} T_{0}$ for all $n \geq 1$

The above discussions lead to the following lemma.

Lemma 3.3 (Iterative lemma). Suppose $f$ and $g$ are real analytic on $D_{0} \times \Pi_{h_{0}}$ and satisfy

$$
\|f\|_{D_{0} \times \Pi_{h_{0}}}+\|g\|_{D_{0} \times \Pi_{h_{0}}} \leq \varepsilon_{0} \leq \min \left\{\frac{(d-1) \pi \gamma}{2 d a \Lambda_{0}|\omega|_{\infty}}, \frac{\pi(1-d q) \gamma}{2 d a \Lambda_{0}|\omega|_{\infty}}, \frac{\gamma b}{2 \Lambda_{0}}\right\}
$$

with $\Lambda_{0}$ sufficiently large. Then for each $n \geq 1$ there exists a parameter and coordinate transformation

$$
\mathfrak{V}_{n}=\mathfrak{U}_{0} \circ \mathfrak{U}_{1} \circ \cdots \circ \mathfrak{U}_{n}: D\left(r_{n}, s_{n}\right) \times \Pi_{h_{n}} \rightarrow D\left(r_{0}, s_{0}\right) \times \in \Pi_{h_{0}}
$$

which transforms the reversible mapping $\mathfrak{M}$ into $\mathfrak{M}_{n}=\mathfrak{V}_{n}^{-1} \mathfrak{M}_{n} \mathfrak{V}_{n}$ such that

$$
\left\|f_{n}\right\|_{D_{n} \times \Pi_{h_{n}}}+\left\|g_{n}\right\|_{D_{n} \times \Pi_{h_{n}}} \leq \varepsilon_{n}=q^{n} \epsilon_{0}
$$

Moreover,

$$
\left\|\mathfrak{V}_{n+1}-\mathfrak{V}_{n}\right\|_{D_{n+1} \times \Pi_{h_{n+1}}} \leq c \Lambda\left(\tau_{n+1}\right) \gamma_{n+1}^{-1} \sigma_{n+1} \varepsilon_{n+1} .
$$

Proof. The estimate of the $f_{n}, g_{n}$ on $D_{n} \times \Pi_{h_{n}}$ follows by applying the KAM step lemma repeatedly. Just note that $\Lambda\left(\tau_{n}\right) \leq \Lambda_{n}$ and

$$
2 \varepsilon_{n} \Lambda_{n} \gamma_{n}^{-1} \leq 2 \varepsilon_{0} \Lambda_{0} \gamma_{0}^{-1} \leq b \quad \text { for all } n \geq 1
$$

In the following the estimate of $\mathfrak{V}_{n}$ is given. By the KAM step, we observe that

$$
\left\|\mathfrak{U}_{n}-\mathrm{id}\right\|_{D_{n} \times \Pi_{h_{n}}} \leq 2 \Lambda\left(\tau_{n}\right) \gamma_{n}^{-1} \sigma_{n} \varepsilon_{n} \quad \text { and } \quad\left\|D \mathfrak{U}_{n}-\operatorname{Id}\right\|_{D_{n} \times \Pi_{h_{n}}} \leq 2 a \Lambda\left(\tau_{n}\right) \gamma_{n}^{-1} \varepsilon_{n}
$$


where $D$ denotes the Jacobian with respect to $(x, z)$.

Then we have

$$
\begin{aligned}
\left\|\mathfrak{V}_{n+1}-\mathfrak{V}_{n}\right\|_{D_{n+1} \times \Pi_{h_{n+1}}} & =\left\|\mathfrak{V}_{n} \circ \mathfrak{U}_{n+1}-\mathfrak{V}_{n}\right\|_{D_{n+1} \times \Pi_{h_{n+1}}} \\
& \leq\left\|D \mathfrak{V}_{n}\right\|_{D_{n} \times \Pi_{h_{n}}}\left\|\mathfrak{U}_{n+1}-\mathrm{id}\right\|_{D_{n+1} \times \Pi_{h_{n+1}}} \\
& \leq c \Lambda\left(\tau_{n+1}\right) \gamma_{n+1}^{-1} \sigma_{n+1} \varepsilon_{n+1}
\end{aligned}
$$

provided that $D \mathfrak{V}_{n}$ is uniformly bounded on $D_{n} \times \Pi_{h_{n}}$.

In fact by induction we have $D \mathfrak{V}_{n}=D \mathfrak{U}_{0} \circ D \mathfrak{U}_{1} \circ \cdots \circ D \mathfrak{U}_{n}$ with the Jacobian evaluated at different points, and

$$
\begin{aligned}
\left\|D \mathfrak{V}_{n}\right\|_{D_{n+1} \times \Pi_{h_{n+1}}} & =\left\|D \mathfrak{U}_{0} \circ D \mathfrak{U}_{1} \circ \cdots \circ D \mathfrak{U}_{n}\right\|_{D_{n+1} \times \Pi_{h_{n+1}}} \leq \prod_{n \geq 0}\left(1+2 a \Lambda\left(\tau_{n}\right) \gamma_{n}^{-1} \varepsilon_{n}\right) \\
& \leq \prod_{n \geq 0}\left(1+\frac{4 a \Lambda_{0} \varepsilon_{0}}{\gamma} \frac{1}{d^{n}}\right) \leq \exp \left\{\frac{4 d a \Lambda_{0} \varepsilon_{0}}{(d-1) \gamma}\right\}
\end{aligned}
$$

which is uniformly bounded and small. Thus this ends the proof of Lemma 3.3 .

\subsection{Convergence of iteration}

We first verify that $r_{n}$ tend to a positive limit and $s_{n}$ tend to 0 . From the definition of $\sigma_{n}, 1-a=e^{-\tau_{n} \sigma_{n}}$ and (3.27) it follows that

$$
\sum_{n \geq 0} \sigma_{n}=\sum_{n \geq 0} \frac{\ln (1-a)^{-1}}{\tau_{n}} \leq \frac{\ln (1-a)}{\ln d q} \int_{\tau_{0}}^{\infty} \frac{\ln \Lambda(t)}{t^{2}} d t .
$$

Hence by choosing $\tau_{0}$ sufficiently large, we can achieve that $\sum_{n \geq 0} \sigma_{n} \leq r / 2$ and thus $r_{n} \rightarrow r_{*} \geq r / 2$ as $n \rightarrow \infty$. From the definition of $\sigma_{n}, \varepsilon_{n}$ and $\sigma_{n} / a<s_{n} \leq \varepsilon_{n}$, it follows that $s_{n} \rightarrow 0$ as $n \rightarrow \infty$.

By Iterative Lemma (see Lemma 3.3), $\mathfrak{V}_{n}$ and $\Omega_{n}(p)$ respectively satisfy

$$
\left\|\mathfrak{V}_{n+1}-\mathfrak{V}_{n}\right\|_{D_{n+1} \times \Pi_{h_{n+1}}} \leq c \Lambda\left(\tau_{n+1}\right) \gamma_{n+1}^{-1} \sigma_{n+1} \varepsilon_{n+1}
$$

and

$$
\left|\Omega_{n+1}(p)-\Omega_{n}(p)\right|_{\Pi_{h_{n}}} \leq a \varepsilon_{n}=a q^{n} \epsilon_{0}
$$

Note that $r_{n} \rightarrow r_{*}, r / 2 \leq r_{*}<r, s_{n} \rightarrow 0, h_{n} \rightarrow 0$ as $n \rightarrow \infty$. Thus the mappings $\mathfrak{V}_{n}$ converge uniformly on

$$
\bigcap_{n \geq 0} D_{n} \times \Pi_{h_{n}}=D_{*} \times \Pi_{\omega, \gamma, \Delta}^{*}, \quad D_{*}=\left\{|\operatorname{Im} \xi|<r_{*}\right\} \times\{0\}
$$


to a mapping $\mathfrak{V}_{*}$ which is real analytic on $D_{*}$ and uniformly continuous on $\Pi_{\omega, \gamma, \Delta}^{*}$. Moreover,

$$
\begin{gathered}
\left\|\mathfrak{V}_{*}-\mathrm{id}\right\|_{D_{*} \times \Pi_{\omega, \gamma}^{*}} \leq \exp \left\{\frac{4 d a \Lambda_{0} \varepsilon_{0}}{(d-1) \gamma}\right\} \frac{2 d \Lambda_{0} \varepsilon_{0} \sigma}{(d-1) \gamma}, \\
\left|\Omega_{*}(p)-\Omega(p)\right|_{\Pi_{\omega, \gamma, \Delta}^{*}} \leq \frac{a \varepsilon_{0}}{1-q}, \\
\left|\langle k, \omega\rangle \frac{\Omega_{*}(p)}{2 \pi}-j\right| \geq \frac{\gamma}{2 \Delta(|k|)} \quad \text { for all } k \in \mathbb{Z}^{m} \backslash\{0\}, j \in \mathbb{Z}, p \in \Pi_{\omega, \gamma, \Delta}^{*} .
\end{gathered}
$$

For the statement of the theorem, we choose $a=19 / 20$ and $b=1 / 35, d=2$, which results in

$$
q \approx \frac{2}{5}, \quad \frac{\ln (1-a)}{\ln d q} \leq 16
$$

\subsection{Estimates of measure for parameters}

In this section the measure of the set $\Pi_{\omega, \gamma, \Delta} \backslash \Pi_{\omega, \gamma, \Delta}^{*}$ of bad parameters is estimated. Recall that by construction, $\Pi_{\omega, \gamma, \Delta}^{*}=\bigcap_{n \geq 0} \Pi_{\omega, \gamma, \Delta}^{n}$, where $\Pi_{\omega, \gamma, \Delta} \supset \Pi_{\omega, \gamma, \Delta}^{0} \supset \Pi_{\omega, \gamma, \Delta}^{1} \supset \cdots$ is a decreasing sequence of closed sets defined inductively during the iteration process by

$$
\Pi_{\omega, \gamma, \Delta}^{n}=\Pi_{\omega, \gamma, \Delta}^{n-1} \backslash \bigcup_{n} R_{\omega, \gamma, \Delta}^{n}, \quad \Pi_{\omega, \gamma, \Delta}^{-1}=\Pi_{\omega, \gamma, \Delta}
$$

with

$$
\mathrm{R}_{\omega, \gamma, \Delta}^{n}=\bigcup_{\substack{\tau_{n-1}<|k| \leq \tau_{n} \\ k \in \mathbb{Z}^{m} \backslash\{0\}, j \in \mathbb{Z}}} \mathrm{R}_{\omega, \gamma, \Delta}^{n, k, j}=\bigcup_{\substack{\tau_{n-1}<|k| \leq \tau_{n} \\ k \in \mathbb{Z}^{m} \backslash\{0\}, j \in \mathbb{Z}}}\left\{p \in \Pi_{\omega, \gamma, \Delta}^{n-1}:\left|\langle k, \omega\rangle \frac{\Omega_{n}(p)}{2 \pi}-j\right|<\frac{\gamma_{n}}{\Delta(|k|)}\right\} .
$$

Lemma 3.4 (Measure estimates). If the Brjuno-Rüssmann approximation function $\Delta$ is chosen such that

$$
\sum_{\ell=1}^{+\infty} \ell^{m}\left(\frac{1}{\ell \Delta(\ell)}\right)^{1 / \lambda}<+\infty
$$

then $\operatorname{meas}\left(\Pi_{\omega, \gamma, \Delta} \backslash \Pi_{\omega, \gamma, \Delta}^{*}\right) \leq c \gamma^{1 / \lambda}$.

Proof. By the proof of Theorem 2.5 and by $\gamma / 2 \leq \gamma_{n} \leq \gamma$, for all $n \geq 0, p \in \mathrm{R}_{\omega, \gamma, \Delta}^{n, k, j}$, we have

$$
\operatorname{meas}\left(\mathrm{R}_{\omega, \gamma, \Delta}^{n, k, j}\right) \leq O\left(\left(\frac{\gamma_{n}}{|k| \Delta(|k|)}\right)^{1 / \lambda}\right) \leq \gamma^{1 / \lambda} O\left(\left(\frac{1}{|k| \Delta(|k|)}\right)^{1 / \lambda}\right)
$$

Next we estimate the measure of the set $\Pi_{\omega, \gamma, \Delta} \backslash \mathrm{R}_{\omega, \gamma, \Delta}^{n}$. Since for $p \in \mathrm{R}_{\omega, \gamma, \Delta}^{n, k, j}$,

$$
\left|\langle k, \omega\rangle \frac{\Omega(p)}{2 \pi}-j\right|<\frac{\gamma_{n}}{\Delta(|k|)},
$$


then we have

$$
|j| \leq|\langle k, \omega\rangle| \frac{\Omega(p)}{2 \pi}+\frac{\gamma_{n}}{\Delta(|k|)} \leq c_{0}|k|,
$$

where $c_{0}$ is a constant independent of $k$. Thus,

$$
\begin{aligned}
\operatorname{meas}\left(\mathrm{R}_{\omega, \gamma, \Delta}^{n}\right) & \leq \sum_{\substack{\tau_{n-1}<|k| \leq \tau_{n} \\
k \in \mathbb{Z}^{m} \backslash\{0\}}} \sum_{\substack{j \in \mathbb{Z} \\
|j| \leq c_{0}|k|}} \operatorname{meas}\left(\mathrm{R}_{\omega, \gamma, \Delta}^{n, k, j}\right) \\
& \leq \gamma^{1 / \lambda} \sum_{\substack{\tau_{n-1<|k| \leq \tau_{n}} \\
k \in \mathbb{Z}^{m} \backslash\{0\}}} \sum_{\substack{j \in \mathbb{Z} \\
|j| \leq c_{0}|k|}} O\left(\left(\frac{1}{|k| \Delta(|k|)}\right)^{1 / \lambda}\right) \\
& \leq \gamma^{1 / \lambda} \sum_{\substack{\tau_{n-1<|k| \leq \tau_{n}} \\
k \in \mathbb{Z}^{m} \backslash\{0\}}} O\left(|k|\left(\frac{1}{|k| \Delta(|k|)}\right)^{1 / \lambda}\right) .
\end{aligned}
$$

Then

$$
\operatorname{meas}\left(\Pi_{\omega, \gamma, \Delta} \backslash \Pi_{\omega, \gamma, \Delta}^{*}\right) \leq \gamma^{1 / \lambda} \sum_{k \in \mathbb{Z}^{m} \backslash\{0\}} O\left(|k|\left(\frac{1}{|k| \Delta(|k|)}\right)^{1 / \lambda}\right) .
$$

Also, by the condition of Lemma 3.4, we have

$$
\begin{aligned}
\sum_{k \in \mathbb{Z}^{m} \backslash\{0\}}|k|\left(\frac{1}{|k| \Delta(|k|)}\right)^{1 / \lambda} & \leq 2^{m} \sum_{\ell=1}^{+\infty} \ell\left(\frac{1}{\ell \Delta(\ell)}\right)^{1 / \lambda}\left(\begin{array}{c}
m+\ell-1 \\
\ell
\end{array}\right) \\
& \leq 2^{2 m-1} \sum_{\ell=1}^{+\infty} \ell^{m}\left(\frac{1}{\ell \Delta(\ell)}\right)^{1 / \lambda}<+\infty
\end{aligned}
$$

Hence

$$
\operatorname{meas}\left(\Pi_{\omega, \gamma, \Delta} \backslash \Pi_{\omega, \gamma, \Delta}^{*}\right) \leq c \gamma^{1 / \lambda}
$$

This completes the proof.

\section{Example and application}

\subsection{Example}

In this subsection we give some examples to discuss the persistence of invariant curves with prescribed frequency and show how to introduce extra parameters in the proof. In the following the non-degenerate condition is even order non-degeneracy. Typically, we only consider the case of second order nondegeneracy for simplicity. The cases of more higher even order can been treated similarly.

Suppose the second order non-degenerate condition is satisfied, i.e., there is some $y^{*}$ such that $\frac{d h}{d y}\left(y^{*}\right)=0$, but $\frac{d^{2} h}{d y^{2}}\left(y^{*}\right) \neq 0$. Without loss of generality we can assume that the 
critical value $y^{*}$ of $h(y)$ is located at the origin. Our initial reversible mapping $\mathcal{A}_{0}$ :

$$
x_{1}=x+\alpha+y^{2}+f(x, y), \quad y_{1}=y+g(x, y),
$$

where the small perturbations $f(x, y)$ and $g(x, y)$ are quasi-periodic in $x$ with frequency $\omega=\left(\omega_{1}, \omega_{2}, \ldots, \omega_{m}\right)$, real analytic in $x, y$ and include the more than 2-order terms of $y$, the variable $y$ has been scaled so that the coefficient of $y^{2}$ equals to 1 . By the method in this paper and KAM iteration with some parameters, we can prove that a family of variant curves can persist under small perturbation, but the frequency $\Omega_{*}(p)$ has some drift. Moreover, the frequency $\Omega_{*}(p)$ satisfies the Brjuno-Rüssmann's non-resonant condition:

$$
\left|\langle k, \omega\rangle \frac{\Omega_{*}(p)}{2 \pi}-j\right| \geq \frac{\gamma}{2 \Delta(|k|)} \quad \text { for all } k \in \mathbb{Z}^{m} \backslash\{0\}, j \in \mathbb{Z} .
$$

\subsection{Application}

In this subsection we will apply the above result to superlinear Duffing's equation

$$
\ddot{x}+x^{3}=f(t)
$$

where $f(t)$ is real analytic and quasi-periodic in $t$ with the frequency $\omega=\left(\omega_{1}, \omega_{2}, \ldots, \omega_{m}\right)$.

Throughout this subsection, we assume $f(t)$ is even in $t$. Introduce a new variable $y=-\dot{x}$, Equation 4.1 is equivalent to the system

$$
\dot{x}=-y, \quad \dot{y}=x^{3}-f(t),
$$

which is reversible with respect to the involution $(x, y) \mapsto(x,-y)$.

Dropping the time-dependent term equation (4.1) becomes

$$
\ddot{x}+x^{3}=0,
$$

which is equivalent to the system

$$
\dot{x}=-y, \quad \dot{y}=x^{3},
$$

which is time-independent Hamiltonian system on $\mathbb{R}^{2}$ :

$$
\dot{x}=-\frac{\partial}{\partial y} h(x, y), \quad \dot{y}=\frac{\partial}{\partial x} h(x, y)
$$

with $h(x, y)=\frac{1}{2} y^{2}+\frac{1}{4} x^{4}$. Clearly, $h$ is positive on $\mathbb{R}^{2}$ except at the only equilibrium point $(x, y)=(0,0)$ of 4.3 where $h=0$. All the solutions of 4.4 are periodic with period tending to zero as $h=E$ tends to infinity.

Suppose $(C(t), S(t))$ is the solution of 4.3 satisfying the initial condition $(C(0), S(0))$ $=(1,0)$. Let $T_{*}>0$ be its minimal period. From 4.3$)$, these analytic functions satisfy 
(i) $C\left(t+T_{*}\right)=C(t), S\left(t+T_{*}\right)=S(t)$ and $C(0)=1, S(0)=0$.

(ii) $\dot{C}(t)=S(t), \dot{S}(t)=-C^{3}(t)$.

(iii) $2 S^{2}(t)+C^{4}(t)=1$.

(iv) $C(-t)=C(t), S(-t)=-S(t)$.

Under the transformation

$$
\Psi: \quad x=c^{1 / 3} \rho^{1 / 3} C\left(\theta T_{*}\right), \quad y=c^{2 / 3} \rho^{2 / 3} S\left(\theta T_{*}\right)
$$

with $c=3 / T_{*}$. The system 4.2 now becomes as follows:

$$
\begin{aligned}
& \dot{\theta}=-\frac{4}{3} d \rho^{1 / 3}+\frac{1}{3} c^{1 / 3} \rho^{-2 / 3} C\left(\theta T_{*}\right) f(t), \\
& \dot{\rho}=-c^{1 / 3} T_{*} \rho^{1 / 3} S\left(\theta T_{*}\right) f(t),
\end{aligned}
$$

$d=c^{4 / 3} / 4$. This system is reversible with respect to $(\rho, \theta) \mapsto(\rho,-\theta)$ and 1-periodic in $\theta$.

As one did in the periodic case, now we change the role of the variable $t$ and $\theta$, and yields that

$$
\begin{aligned}
& \frac{d t}{d \theta}=\left[-\frac{4}{3} d \rho^{1 / 3}+\frac{1}{3} c^{1 / 3} \rho^{-2 / 3} C\left(\theta T_{*}\right) f(t)\right]^{-1}, \\
& \frac{d \rho}{d \theta}=-c^{1 / 3} T_{*} \rho^{1 / 3} S\left(\theta T_{*}\right) f(t)\left[-\frac{4}{3} d \rho^{1 / 3}+\frac{1}{3} c^{1 / 3} \rho^{-2 / 3} C\left(\theta T_{*}\right) f(t)\right]^{-1},
\end{aligned}
$$

this system is reversible with respect to the involution $(\rho, t) \mapsto(\rho,-t)$ and 1-periodic in the new time variable $\theta$.

Our next goal is to obtain asymptotic expansions for $t_{1}$ and $\rho_{1}$. For $\rho_{0}$ large enough, the second equation of 4.5 can be rewritten as

$$
\frac{d \rho}{d \theta}=\frac{3 T_{*} c^{1 / 3}}{4 d} S\left(\theta T_{*}\right) f(t)+O\left(\rho^{-1}\right) .
$$

An integration of this equation leads to

$$
\rho(\theta)=\rho_{0}+O(1), \quad \theta \in[0,1] .
$$

Therefore,

$$
\rho(\theta)^{-1 / 3}=\rho_{0}^{-1 / 3}\left(1+\rho_{0}^{-1} O(1)\right)^{-1 / 3} .
$$

Expanding $\left(1+\rho_{0}^{-1} O(1)\right)^{-1 / 3}$ yields

$$
\rho(\theta)^{-1 / 3}=\rho_{0}^{-1 / 3}+O\left(\rho_{0}^{-4 / 3}\right), \quad \theta \in[0,1]
$$


for $\rho_{0}$ large enough, by 4.7 and the first equality of 4.5, we get

$$
\frac{d t}{d \theta}=O\left(\rho_{0}^{-1 / 3}\right), \quad \theta \in[0,1]
$$

which implies that

$$
t(\theta)=t_{0}+O\left(\rho_{0}^{-1 / 3}\right), \quad \theta \in[0,1]
$$

for $\rho_{0}$ large enough. Substituting (4.6-4.8 into the second equality of 4.5), we have, for $\theta \in[0,1]$,

$$
\frac{d \rho}{d \theta}=\frac{3 T_{*} c^{1 / 3}}{4 d} S\left(\theta T_{*}\right) f\left(t_{0}\right)+O\left(\rho_{0}^{-1 / 3}\right)
$$

for $\rho_{0}$ large enough. An integration of 4.9 over $\theta \in[0,1]$ yields

$$
\rho_{1}=\rho_{0}+O\left(\rho_{0}^{-1 / 3}\right)
$$

for $\rho_{0}$ large enough, where $\rho_{1}=\rho(1)$. Substituting 4.7 and 4.8 into the first equality of 4.5, we have, for $\theta \in[0,1]$,

$$
\frac{d t}{d \theta}=-\frac{3}{4 d} \rho_{0}^{-1 / 3}+O\left(\rho_{0}^{-4 / 3}\right)
$$

for $\rho_{0}$ large enough. An integration 4.10 over $\theta \in[0,1]$ yields

$$
t_{1}=t_{0}-\frac{3}{4 d} \rho_{0}^{-1 / 3}+O\left(\rho_{0}^{-4 / 3}\right)
$$

where $t_{1}=t(1)$.

To apply the invariant curve theorem obtained in Liu [17], introduce a new variable $\mu$ by

$$
\delta \mu=-\frac{3}{4 d} \rho^{-1 / 3}, \quad \mu \in[-2,-1] .
$$

Obviously, $\rho \gg 1 \Longleftrightarrow \delta \ll 1$. Under this change of variable, we may get an expression of time-1 map of (4.5):

$$
\mathcal{P}:\left\{\begin{array}{l}
t_{1}=t_{0}+\delta \mu_{0}+\delta \widetilde{f}_{1}\left(t_{0}, \mu_{0}, \delta\right), \\
\mu_{1}=\mu_{0}+\delta \widetilde{f}_{2}\left(t_{0}, \mu_{0}, \delta\right),
\end{array}\right.
$$

where $\tilde{f}_{1}, \tilde{f}_{2}$ are real analytic and quasi-periodic in $t_{0}$ with the frequency $\omega=\left(\omega_{1}, \omega_{2}, \ldots\right.$, $\left.\omega_{m}\right)$ and $\widetilde{f}_{1}, \widetilde{f}_{2} \rightarrow 0$ as $\delta \rightarrow 0$. If $\delta$ is sufficiently small, therefore the assumptions of Theorem 2 in [17] are met. Hence the existence of the invariant curves of $\mathcal{P}$ is guaranteed by Theorem 2 in [17], the invariant curves are real analytic quasi-periodic with the frequency $\omega=\left(\omega_{1}, \omega_{2}, \ldots, \omega_{m}\right)$. Then system (4.1) has infinitely many quasi-periodic solutions with frequencies $\left\{\omega=\left(\omega_{1}, \omega_{2}, \ldots, \omega_{m}\right), \frac{1}{\delta \alpha}\right\}$. 
As an application of the invariant curve theorem obtained in this paper, we shall study the existence of quasi-periodic solutions whose frequencies may be different from $\left\{\omega=\left(\omega_{1}, \omega_{2}, \ldots, \omega_{m}\right), \frac{1}{\delta \alpha}\right\}$ for system 4.1. To apply the theorem obtained in Section 2 , introduce a new variable $\mu$ by

$$
\mu^{2}=\frac{3}{4 d} \rho^{-1 / 3}
$$

Under this change of variable, we may get an expression of time-1 map of (4.5):

$$
P:\left\{\begin{array}{l}
t_{1}=t_{0}-\mu_{0}^{2}+\widetilde{f}_{1}\left(t_{0}, \mu_{0}\right) \\
\mu_{1}=\mu_{0}+\widetilde{f}_{2}\left(t_{0}, \mu_{0}\right)
\end{array}\right.
$$

where $\widetilde{f}_{1}, \widetilde{f}_{2}$ are real analytic and quasi-periodic in $t_{0}$ with the frequency $\omega=\left(\omega_{1}, \omega_{2}, \ldots\right.$, $\left.\omega_{m}\right)$ and $\widetilde{f}_{1}=O\left(\mu_{0}^{8}\right), \widetilde{f}_{2}=O\left(\mu_{0}^{9}\right)$.

Since the change of variables 4.11 commutes with the involution $\mathcal{R}:(\mu, t) \mapsto(\mu,-t)$, hence $P$ is also reversible with respect to the same involution $\mathcal{R}$. Let $\mu=z+p$, the above mapping $P$ becomes

$$
\widetilde{P}:\left\{\begin{array}{l}
t_{1}=t_{0}-p^{2}+\widetilde{f}_{1}\left(t_{0}, z_{0} ; p\right) \\
z_{1}=z_{0}+\widetilde{f}_{2}\left(t_{0}, z_{0} ; p\right)
\end{array}\right.
$$

where $\widetilde{f}_{1}\left(t_{0}, z_{0} ; p\right)=-z_{0}^{2}-2 z_{0} p+\widetilde{f}_{1}\left(t_{0}, z_{0}+p\right), \widetilde{f}_{2}\left(t_{0}, z_{0} ; p\right)=\widetilde{f}_{2}\left(t_{0}, z_{0}+p\right), \mu_{0} \in(-\delta, \delta)$, $z_{0} \in(-\widetilde{\delta}, \widetilde{\delta}), p \in(0, \delta-\widetilde{\delta})$ or $p \in(-\delta+\widetilde{\delta}, 0), \delta>\widetilde{\delta}>0$. Now we apply Theorem 2.7 to prove the existence of quasi-periodic solutions and the boundedness of all solutions for (4.1).

\subsection{The main result}

Theorem 4.1. Every solution of 4.1) with a real analytic quasi-periodic function $f(t) \in$ $Q_{r}(\omega), \omega$ satisfying the non-resonance condition (2.1) is bounded. Moreover, (4.1) has infinitely many quasi-periodic solutions.

Proof. Since $\Omega(p)=-p^{2}$, and $\Omega^{(2)}(p)=-2 \neq 0$ when $p \in(0, \delta-\widetilde{\delta})$ or $p \in(-\delta+\widetilde{\delta}, 0)$. Moreover, by Theorem 2.5 there exist Brjuno-Rüssmann approximation function $\Delta$ such that

$$
\sum_{\ell=1}^{+\infty} \frac{\ell^{m-1}}{\Delta(\ell)}<+\infty
$$

Hence $\Pi_{\omega, \gamma, \Delta}$ the set of all $p \in(0, \delta-\widetilde{\delta})$ or $p \in(-\delta+\widetilde{\delta}, 0)$ satisfying

$$
\left|\langle k, \omega\rangle \frac{-p^{2}}{2 \pi}-j\right| \geq \frac{\gamma}{\Delta(|k|)} \quad \text { for all } k \in \mathbb{Z}^{m} \backslash\{0\}, j \in \mathbb{Z}
$$


has positive measure. Then from Theorem 2.7, we know that the map $\widetilde{P}$ has invariant curves if $\delta>0$ are sufficiently small. If the conditions of Theorem 4.1 hold and $\delta>0$ are sufficiently small, therefore the assumptions of Theorem 2.7 are met. Hence the existence of the invariant curves of $\widetilde{P}$ is guaranteed by Theorem 2.7, the invariant curves are real analytic quasi-periodic with the frequency $\omega=\left(\omega_{1}, \omega_{2}, \ldots, \omega_{m}\right)$. Undoing the change of variables we obtain the invariant curves of $P$. Then system (4.1) has infinitely many quasi-periodic solutions as well as the boundedness of solutions.

Remark 4.2. It follows from the proof of Theorem 4.1 that if the conditions of Theorem 4.1 hold, then system 4.1 has infinitely many quasi-periodic solutions with frequencies $\{\omega=$ $\left.\left(\omega_{1}, \omega_{2}, \ldots, \omega_{m}\right), \frac{1}{-p^{2}}\right\}$ satisfying the following non-resonance condition

$$
\begin{gathered}
\left|\langle k, \omega\rangle \frac{-p^{2}}{2 \pi}-j\right| \geq \frac{\gamma}{\Delta(|k|)} \quad \text { for all } k \in \mathbb{Z}^{m} \backslash\{0\}, j \in \mathbb{Z}, \\
p \in(0, \delta-\widetilde{\delta}) \quad \text { or } \quad p \in(-\delta+\widetilde{\delta}, 0), \quad \delta>\widetilde{\delta}>0
\end{gathered}
$$

and $\delta$ is sufficiently small.

\section{References}

[1] L. Carbajal, D. del-Castillo-Negrete and J. J. Martinell, Dynamics and transport in mean-field coupled, many degrees-of-freedom, area-preserving nontwist maps, Chaos 22 (2012), 013137, 15 pp.

[2] C. Cheng and Z. Xia, KAM theory without the twist condition, in: Dynamical Systems, edited by Y. Jiang, L. Wen, Proceedings of the international conference in honor of Professor Liao Shantao (1999), 16-22.

[3] D. B. A. del Castillo Negrete, Dynamics and Transport in Rotating Fluids and Transition to Chaos in Area-preserving Nontwist Maps, Thesis (Ph.D.), The University of Texas at Austin, 1994.

[4] D. del-Castillo-Negrete, J. M. Greene and P. J. Morrison, Area preserving nontwist maps: Periodic orbits and transition to chaos, Phys. D 91 (1996), no. 1-2, 1-23.

[5] _ Renormalization and transition to chaos in area preserving nontwist maps, Phys. D 100 (1997), no. 3-4, 311-329.

[6] A. Delshams and R. de la Llave, KAM theory and a partial justification of Greene's criterion for nontwist maps, SIAM J. Math. Anal. 31 (2000), no. 6, 1235-1269.

[7] R. Dieckerhoff and E. Zehnder, Boundedness of solutions via the twist-theorem, Ann. Scuola Norm. Sup. Pisa. Cl. Sci. (4) 14 (1987), no. 1, 79-95. 
[8] A. González-Enríquez, A. Haro and R. de la Llave, Singularity theory for non-twist KAM tori, Mem. Amer. Math. Soc. 227 (2014), no. 1067, 115 pp.

[9] M.-R. Herman, Sur les courbes invariantes par les difféomorphismes de l'anneau I, Astérisque 103-104, Société Mathématique de France, Paris, 1983.

[10] Sur les courbes invariantes par les difféomorphismes de l'anneau II, Astérisque 144 (1986), 248 pp.

[11]_, Talk at the conference on dynamical systems, Oberwolfach, 1997.

[12] P. Huang, X. Li and B. Liu, Quasi-periodic solutions for an asymmetric oscillation, Nonlinearity 29 (2016), no. 10, 3006-3030.

[13] Invariant curves of smooth quasi-periodic mappings, Discret. Contin. Dyn. Syst. 38 (2018), no. 1, 131-154.

[14] M. Levi, Quasiperiodic motions in superquadratic time-periodic potentials, Comm. Math. Phys. 143 (1991), no. 1, 43-83.

[15] M. Levi and J. Moser, A Lagrangian proof of the invariant curve theorem for twist mappings, in: Smooth Ergodic Theory and its Applications (Seattle, WA, 1999), 733746, Proc. Sympos. Pure Math. 69, Amer. Math. Soc., Providence, RI, 2001.

[16] J. E. Littlewood, Some Problems in Real and Complex Analysis, D. C. Heath and COmpany Raytheon Education Company, Lexington, Mass., 1968.

[17] B. Liu, Invariant curves of quasi-periodic reversible mappings, Nonlinearity 18 (2005), no. 2, 685-701.

[18] B. Liu and J. J. Song, Invariant curves of reversible mappings with small twist, Acta Math. Sin. (Engl. Ser.) 20 (2004), no. 1, 15-24.

[19] B. Liu and J. You, Quasiperiodic solutions of Duffing's equations, Nonlinear Anal. 33 (1998), no. 6, 645-655.

[20] G. R. Morris, A case of boundedness in Littlewood's problem on oscillatory differential equations, Bull. Austral. Math. Soc. 14 (1976), no. 1, 71-93.

[21] J. Moser, On invariant curves of area-preserving mappings of an annulus, Nachr. Akad. Wiss. Göttingen Math.-Phys. Kl. II 1962 (1962), 1-20.

[22] _ Combination tones for Duffing's equation, Comm. Pure Appl. Math. 18 (1965), 167-181. 
[23] _ Stable and Random Motions in Dynamical Systems, N. J. Annals of Mathematics Studies 77, Princeton University Press, Princeton, N. J., 1973.

[24] A stability theorem for minimal foliations on a torus, Ergodic Theory Dynam. Systems 8 (1988), Charles Conley Memorial Issue, 251-281.

[25] J. Pöschel, A lecture on the classical KAM theorem, in: Smooth Ergodic Theory and its Applications (Seattle, WA, 1999), 707-732, Proc. Sympos. Pure Math. 69, Amer. Math. Soc., Providence, RI, 2001.

[26] _ _ KAM à la R, Regul. Chaotic Dyn. 16 (2011), no. 1-2, 17-23.

[27] H. Rüssmann, Kleine Nenner I: Über invariante Kurven differenzierbarer Abbildungen eines Kreisringes, Nachr. Akad. Wiss. Göttingen Math.-Phys. Kl. II 1970 (1970), 67105.

[28] _ KAM iteration with nearly infinitely small steps in dynamical systems of polynomial character, Discrete Contin. Dyn. Syst. Ser. S 3 (2010), no. 4, 683-718.

[29] M. B. Sevryuk, Reversible Systems, Lecture Notes in Mathematics 1211, SpringerVerlag, Berlin, 1986.

[30] C. L. Siegel and J. K. Moser, Lectures on Celestial Mechanics, Classics in Mathematics, Springer-Verlag, Berlin, 1995.

[31] C. Simó, Invariant curves of analytic perturbed nontwist area preserving maps, Regul. Chaotic Dyn. 3 (1998), no. 3, 180-195.

[32] J. Xu, J. You and Q. Qiu, Invariant tori for nearly integrable Hamiltonian systems with degeneracy, Math. Z. 226 (1997), no. 3, 375-386.

[33] D. Zhang and J. Xu, Invariant curves of analytic reversible mappings under BrjunoRüssmann's non-resonant condition, J. Dynam. Differential Equations 26 (2014), no. 4, 989-1005.

[34] V. Zharnitsky, Invariant curve theorem for quasiperiodic twist mappings and stability of motion in the Fermi-Ulam problem, Nonlinearity 13 (2000), no. 4, 1123-1136.

Peng Huang

School of Mathematics and Statistics, Guizhou University of Finance and Economics, Guiyang 550025, China

E-mail address: hp@mail.bnu.edu.cn 